\title{
Flexible transparent conductive materials based on silver nanowire networks:
}

\section{a review}

(Ed: Editor20)

Q.1 Daniel Langley ${ }^{1,2}$, Gaël Giusti ${ }^{1}$, Céline Mayousse ${ }^{3}$, Caroline Celle $^{3}$, Daniel Bellet $^{1}$ and Jean-Pierre Simonato ${ }^{3}$

\footnotetext{
${ }^{1}$ Laboratoire des Matériaux et du Génie Physique, CNRS-Grenoble INP, 3 Parvis Louis Néel, F-38016 Grenoble, France

${ }^{2}$ Laboratoire de Physique des Solides, Interfaces et Nanostructures, Allée du 6 Août, 17 (Bâtiment B5a) Sart Tilman, B-4000 Liège 1, Belgium

${ }^{3}$ CEA-Liten, DTNM, CRE, 17 rue des Martyrs, F-38054 Grenoble Cedex 9, France

E-mail: jean-pierre.simonato@cea.fr
}

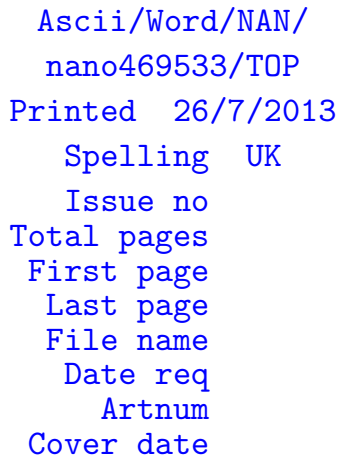

Received 10 April 2013, in final form 5 July 2013

Published

Online at stacks.iop.org/Nano/24/000000

\begin{abstract}
The class of materials combining high electrical or thermal conductivity, optical transparency and flexibility is crucial for the development of many future electronic and optoelectronic devices. Silver nanowire networks show very promising results and represent a viable alternative to the commonly used, scarce and brittle indium tin oxide. The science and

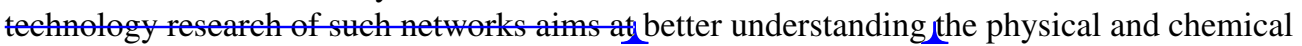
properties of this nanowire-based material while opening attractive new applications.
\end{abstract}

Q.2 (Some figures may appear in colour only in the online journal)

\section{Contents}

1. Introduction

2. Fabrication of silver nanowires

3. Fabrication of silver nanowire networks

4. Properties of TCM based on silver nanowire networks 4.1. Electrical properties

4.2. Optical properties

4.3. Optimizing the electro-optical properties

4.4. Thermal properties

4.5. Structural and mechanical properties

5. Simulation appreaches of the physical properties of metallic nanowire networks

5.1. Simulation of electrical properties

5.2. Simulation of the optical properties

6. Applications

6.1. Solar cells
6.2. Transparent heaters 12

6.3. Displays (OLED) 13

6.4. Touch panels 13

7. Closing remarks and future work 14

Acknowledgments 15

References 15

\section{Introduction}

Thin films with a combination of high electrical conductivity and optical transparency are crucial for many modern devices such as touch panels, e-papers, organic light-emitting diodes (OLEDs), liquid-crystal displays (LCDs), and solar cells. In the case of solar cells, the transparent electrode works as $\_$anode for extracting separated charge carriers from the absorbing layer, while for OLEDs, they inject carriers. The properties of these fabricated devices continue to develop intensively, expanding the industrial demand for transparent 
Table 1. Main property requirements of a transparent electrode for some important applications. Plus signs indicate better values.

\begin{tabular}{llll}
\hline Application & Transparency & Conductivity & Flexibility \\
\hline Photovoltaics & +++ & +++ & + \\
OLED/PLED & ++ & ++ & + \\
Film heaters & + & +++ & ++ \\
Touch screens & +++ & ++ & ++ \\
\hline
\end{tabular}

electrodes. Therefore numerous scientific investigations have been devoted to explore different possibilities for the fabrication of thin layers exhibiting good compromise between optical transparency and electrical conductivity. The development of new transparent conductive materials is mostly dictated by the requirements of the specific application, however low electrical resistivity and low optical absorption always constitute significant prerequisites. The mainstream of scientific studies and current industrial technologies mainly rely on vacuum processes and transparent conductive oxides (TCOs) such as indium tin oxide (ITO), fluorine doped tin oxide (FTO) or aluminium-doped zinc oxide (AZO) thin films. The next generation of optoelectronic devices will require conductive electrodes which are flexible, cheap and compatible with large-scale manufacturing methods [1].

However, needs are application dependent thus it is not possible to give an overall set of ideal figures of merits for emerging flexible transparent electrodes. For instance, significant light scattering (as measured by the haze factor) can be valuable for photovoltaics but is detrimental when electrodes are used for touch panel displays. Many parameters have to be taken into account for each specific application. Cost, optical properties, thermal stability, processability, chemical compatibility with other materials and work function are only a few of the key parameters. Three main criteria are presented in table 1 with expected performance for some applications. Transparency, electrical conductivity and mechanical flexibility are among the most important combined properties achieved with silver nanowire $(\mathrm{AgNW})$ based electrodes. Even for these principal properties, different applications require different specifications.

The indications provided in table 1 are meant as a general guide to the average requirements of a stated application family. With respect to each property there is a broad range of requirements depending on the type of application that is being considered.

Many potential applications have been recently demonstrated with this new material, like electromagnetic interference shielding [2,3], radio frequency antenna [4-6] and others. However, this review will essentially focus on applications which will find outstanding improvements thanks to the three aforementioned properties of flexible electrodes based on random networks of AgNWs.

While TCOs are very well adapted for some applications, they suffer from significant limitations such as cost of fabrication process, scarcity (especially concerning indium based TCOs like ITO) and brittleness. Hence a variety of other materials have been intensively investigated recently: carbon nanotube networks (CNTs), graphene thin films, conducting polymers, metallic grids and metal nanowire networks [1, 7-9]. When selecting the appropriate material for an application it is crucial to consider the costs associated with the raw materials and production methods used to fabricate the electrode, and whether high-temperature or vacuum processes are required for, or incompatible with, device production and the specific needs of the application.

Table 2 provides a brief summary of some fabrication methods of electrodes using transparent conductive materials (TCMs) - it is by no means exhaustive, but allows a simple comparison of the currently available and emerging materials. The processing temperatures reported in table 2 refer to the ranges of temperatures that the film may be exposed to during device fabrication. The lower temperature refers to the lowest temperature that electrodes can be fabricated at and the upper value is related to the stability of the material. Each type of TCM has strengths and weaknesses and in the end the material to use must be determined by the needs of the application.

A brief comment on the uniformity of graphene films is needed. For low-cost production of graphene films, graphene flakes are exfoliated from highly oriented pyrolytic graphite (HOPG). Films produced with these flakes are low cost but have poor uniformity and high resistances.

Regarding the cost identified in table 2, there are a broad range of factors considered: the cost of the raw materials and also processing costs required for electrode fabrication. Each material can produce an electrode at low cost, but the quality of such electrodes varies. The costs indicated in the table refer to the costs associated with the best-quality electrodes-those of highest optical transmission with the lowest electrical resistance. There are a range of costs associated with TCOs due to the broad variety of available materials in this family. An in-depth cost analysis of ITO, CNTs and AgNWs has been performed by Emmott et al [34].

The present review focuses on the AgNW networks which can be produced by low-cost solution-based methods and exhibit very promising properties. We will briefly overview the fabrication of the nanowires in solution and their arrangement into networks, their main physical properties and the prospects for future studies and improvements. Some time is also spent discussing the application of this material to devices providing potential to introduce nanoscience and nanotechnology into everyday life (screens, solar cells) and open up new scientific opportunities.

\section{Fabrication of silver nanowires}

The synthesis of one-dimensional nanomaterials has grown impressively in the last two decades. In particular, the fabrication of metallic nanowires has been tackled recently for the development of transparent flexible electrodes. One of the very important advanced arguments is the need to obtain materials available from low-cost solution-based processes. Among possible metallic nanostructures, AgNWs have been the most studied for several reasons. Bulk silver has the highest electrical conductivity and thus has been the focus of much research. Moreover, synthesis of AgNWs 
Table 2. General guide to transparent conductive material electrode fabrication.

\begin{tabular}{|c|c|c|c|c|}
\hline Fabrication method $^{\mathrm{a}}$ & TCOs & Graphene & CNTs & AgNWs \\
\hline Sputtering & +++ & - & - & - \\
\hline Spray deposition & ++ & + & +++ & +++ \\
\hline Screen printing & -- & + & ++ & ++ \\
\hline Uniformity & +++ & + to +++ & ++ & ++ \\
\hline Typical thickness (nm) & $100-300$ & $<5$ & $<10$ & $25>600^{\mathrm{d}}$ \\
\hline Typical $R_{\mathrm{s}}(\Omega / \square)$ & $5-100$ & $30-5000$ & $60-300$ & $1-50$ \\
\hline Typical transmission (at $550 \mathrm{~nm}$ ) & $80-97 \%$ & $80-96 \%$ & $80-91 \%$ & $80-96 \%$ \\
\hline Typical references & [9-13] & [14-19] & {$[11,20-26]$} & {$[27-33]$} \\
\hline
\end{tabular}

a Fabrication method refers to the production of electrodes directly, not to the production of the constituent components which are used to fabricate the network.

${ }^{b}$ Deposition temperatures of TCOs usually require several hundred degrees celsius or the use of vacuum processes such as sputtering.

${ }^{\mathrm{c}}$ Networks can be fabricated at room temperature (RT) but if device fabrication requires high temperature processes vacuum or encapsulation are required to stabilize the films.

d Thickness is diameter and density dependent though with high mechanical pressure it can be reduced to the diameter of a single nanowire [31].

is rather easy whereas protocols for large-scale synthesis of nanowires with other metals are still missing. However, a few other metallic nanowires have been prepared, and some of them have shown interesting properties for the fabrication of electrically active transparent conductors. In particular, copper [35-38], gold [39, 40] and cupronickel [41] nanowires have demonstrated promising results. To date, the most studied metallic nanowires are made of silver.

Several routes have been followed experimentally to fabricate silver nanostructures with various shapes and form factors. These methods include the hydrothermal method, microwave-assisted process, electrochemical technique, UV irradiation technique, template technique (see Coskun et al [42], Chen et al [43] and references therein). When compared to these methods, the polyol approach appears as the most promising synthetic procedure regarding ease of mass production, cost and simplicity. This method proposes a simple route for the synthesis of metal nanoparticles through reduction of metallic salts by a polyol, generally ethylene glycol $[44,45]$. Crystallization of noble metals usually leads to highly symmetric cubic unit cells. Thus, to obtain the growth of nanowires from solutions of isotropic nanoparticles, anisotropic confinement must be applied in order to achieve unidimensional growth [46]. The method of fabrication of AgNWs was first developed by the team of Xia [47, 48]. Ethylene glycol reduces the metal precursor (usually silver nitrate) in the presence of a nucleating agent and polyvinyl pyrrolidone (PVP). The PVP plays the role of capping agent capable of controlling the growth rates of different surfaces of silver nanospecies. The near-unanimous choice of ethylene glycol as a reducer is based on its ability to solvate both $\mathrm{AgNO}_{3}$ and PVP combined with its high boiling point $\left(196^{\circ} \mathrm{C}\right)$ necessary for the realization of syntheses at high temperature.

The exact mechanism of $\mathrm{AgNW}$ growth is complex and has not been entirely clarified. First, ethylene glycol reduces $\mathrm{Ag}^{+}$ions in $\mathrm{Ag}$ atoms, thereby inducing silver crystal formation and subsequently growth of silver nanostructures. The synthesis of AgNWs has therefore three distinct steps, nucleation, evolution of nuclei into seeds and growth of seeds into nanocrystals. In these steps the phenomena of atomic addition and aggregate formation are essentially governed by mechanisms of diffusion and surface energy minimization. By modifying the thermodynamics and kinetics of each of these steps, it is possible to have some control on the form of the synthesized nanocrystals [49]. Different approaches have been explored. The addition of particles playing the role of seeds can change the process of nucleation. Various nucleating agents, either in a pure form or as combination of a few salts, have been used to study the effect on AgNW growth [43, 48, 50-52]. In general, chlorinated derivatives give good results, and in particular $\mathrm{NaCl}$ is often preferred. The presence of a nucleating agent directly affects the morphology of initially formed metallic seeds. Thus, the addition of $\mathrm{NaCl}$ leads to the formation of $\mathrm{AgCl}$ nanocrystallites and reduces the concentration of free cationic silver ions in the solution during initial seed formation. These slow reaction conditions enable the formation of the thermodynamically more stable multiply-twinned Ag seeds required for wire growth. The seed structures have an important role in the shape evolution of metal nanocrystals and therefore in the resulting materials. It has also been demonstrated that the addition of traces of salts involves selective oxidative etching controlling the structure and population of seeds once formed. Ag nanocubes or AgNWs can be obtained selectively just by modifying salt amounts [53-55]. These parameters are key for the fabrication of one-dimensional $\mathrm{Ag}$ nanostructures. According to Xia et al [47, 49, 56] the nanowires are derived from multiple-twinned particles. Among the various possible seed structures (crystal, simple twin or multiple twins), multiply-twinned decahedra provide the most thermodynamically stable seed, as it is bound almost 

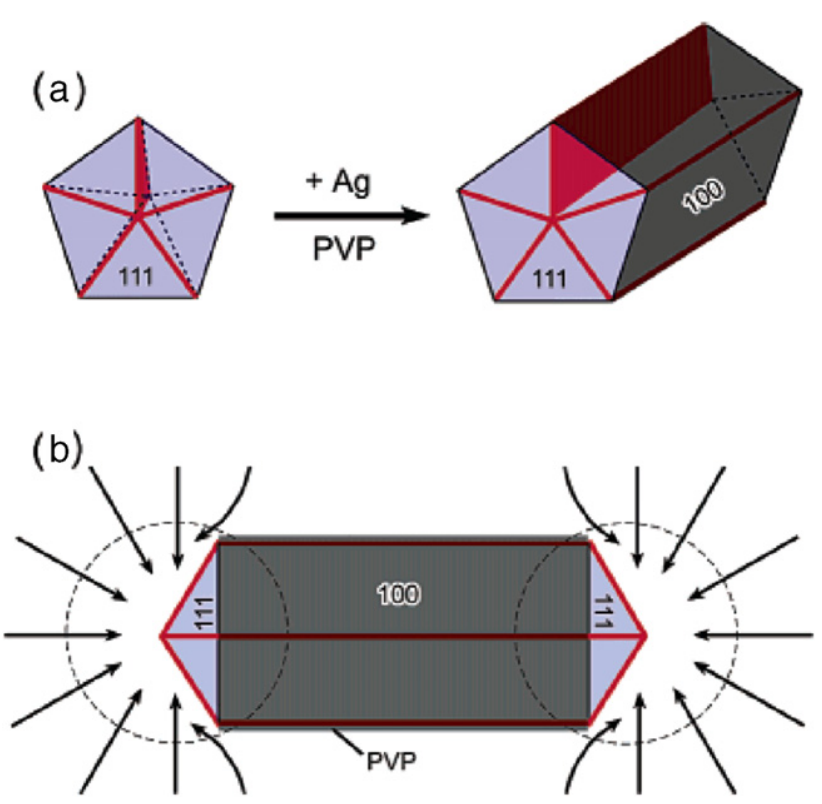

Figure 1. Schematic illustration of the mechanism proposed to account for the growth of AgNWs with pentagonal cross sections: (a) evolution of a nanorod from a multiply-twinned nanoparticle (MTP) of silver under the confinement of five twin planes and with the assistance of PVP. The ends of this nanorod are terminated by $\{111\}$ facets, and the side surfaces are bounded by $\{100\}$ facets. The strong interaction between PVP and the $\{100\}$ facets is indicated with a dark-grey colour, and the weak interaction with the $\{111\}$ facets is marked by a light-blue colour. The red lines on the end surfaces represent the twin boundaries that can serve as active sites for the addition of silver atoms. The plane marked in red shows one of the five twin planes that can serve as the internal confinement for the evolution of nanorods from MTP. (b) Schematic model illustrating the diffusion of silver atoms towards the two ends of a nanorod, with the side surfaces completely passivated by PVP. This drawing shows a projection perpendicular to one of the five side facets of a nanorod, and the arrows represent the diffusion fluxes of silver atoms. Reprinted with permission from [46], copyright 2013 American Chemical Society.

entirely by the lower energy $\{111\}$ facets. It is therefore the most naturally abundant seed but also the most reactive because of the presence of twin defects. Sun et al [57] have recently reported that each nanowire is composed of a heavily strained core encapsulated in a less-strained sheath. The crystalline defects where the cores are exposed provide active sites for growing the nanowires along their longitudinal axes, while the less-strained side surfaces of the nanowires, bound by $\{100\}$ facets, have lower reactivity towards the deposition of silver atoms (see figure 1).

Once the pentagonal rod is formed, PVP plays a key role in the growth mechanism. Originally used to prevent the aggregation of nanoparticles, it has also proven to be an agent controlling the structure of the nano-objects. Indeed, it reacts preferentially with the $\{100\}$ facets of the silver nanoparticles, through interactions between surface silver atoms and the oxygen atoms of the PVP carboxyl groups [58]. Tests performed with other polymers like poly(ethyleneoxide) (PEO) or polyvinylalcohol (PVA) did not lead to satisfying results [48]. Concerning the experimental protocol of the synthesis, many parameters have to be extremely well controlled, which is a common feature for the synthesis of nanoparticles. A recently published parametric study gives some details related to the effects of temperature, injection rate, molar ratio of PVP to silver, $\mathrm{NaCl}$ amount and stirring rate [42]. All of these parameters are important and for some of them tiny changes can lead to dramatic alterations of the nanoparticle's size and shape. It should also be emphasized that the quality of chemical reagents is extremely important, in particular the purity of the ethyleneglycol. A few grams of AgNWs can be obtained through a single experiment which allows large area deposition considering that only few tens of $\mathrm{mg}$ are deposited per $\mathrm{m}^{2}$ to obtain a $\sim 90 \%$ transparency [59]. The nanowires have generally diameters in the order of a few tens of $\mathrm{nm}$ and lengths of several $\mu \mathrm{m}$, resulting in a form factor (length/diameter ratio) typically in the 50-500 range (see figure 2).

Recently, very long AgNWs have been synthesized thanks to a successive multistep growth method. Very high-aspect ratio of 1000-3000 with length of over $300 \mu \mathrm{m}$ and diameter of less than $150 \mathrm{~nm}$ were obtained [60].

After synthesis, the as-obtained product requires some purification. Indeed, a mixture of nanowires and nanoparticles in organic solvent is generally obtained. To remove most of the ethylene glycol and excess PVP, several washes with acetone are commonly used. To separate the nanoparticles from the nanowires the product solution is put through a centrifuge. According to different research groups, the parameters of the commonly used centrifugation procedure can be changed from $20 \mathrm{~min}$ at $2000 \mathrm{rpm}$ [48, 50, 61], $6000 \mathrm{rpm}$ [42] or $8000 \mathrm{rpm}$ [62] to $10 \mathrm{~min}$ at $16000 \mathrm{rpm}$ [63]. Most of the time the product is then dispersed in another solvent such as water [6, 42, 62-64], ethanol [42, 50] or methanol [65] to be washed a second time. Filtration has also been described to further purify the product, either by filtering through a polycarbonate membrane with $0.8 \mu \mathrm{m}$ pore size $[51,58]$ or a glass filter with mean pore size of $5-40 \mu \mathrm{m}$ $[66,67]$. Although less common, cross-flow filtration is also a possibility [68]. Another very straightforward method is to let the crude reaction mixture settle for few days before removing supernatant. This decantation technique allows easy production of a high quantity of nanowires in a single run [69].

\section{Fabrication of silver nanowire networks}

The development of high-performance transparent conductive material increasingly requires the use of nanostructures. Therefore, it is necessary to develop simple, reliable and cost-efficient fabrication techniques to achieve both random and ordered nanowire arrays. Assembling such networks is also a critical step to control their resulting electro-optical properties. In respect to their physical properties and ease of synthesis (see section 2), high-aspect-ratio metallic nanowires are one of the most attractive materials to fabricate such networks [70].

Random nanowire networks can be readily and cheaply made by solution-process techniques such as spray coating $[22,29,30,66]$, drop casting $[27,71,72]$, spin coating $[73,74]$ or rod-coating [75]. All these techniques are 

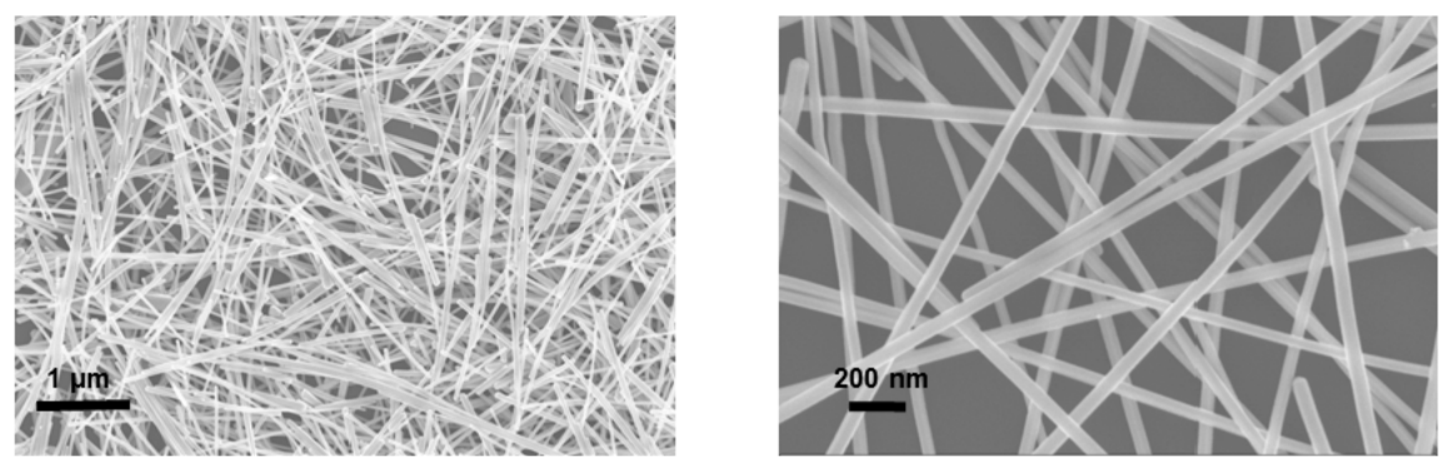

Figure 2. Scanning electron microscopy (SEM) images of silver nanowires at two magnifications.

compatible with very low temperature deposition processes and do not necessitate any vacuum equipment. On top of that, a technique such as spray coating is easily surface scalable. Such solution-processed electrodes have already demonstrated their ability to be integrated into operational devices such as organic solar cells [27, 74].

Nevertheless, while working with colloidal solutions and then depositing them as thin films, it is often difficult to achieve high reproducibility. It is well known that drop cast nanowire networks may show spatial inhomogeneities known as 'coffee ring effect' on the substrates during the solvent evaporation step [75, 76]. Films obtained from air-spraying coatings are usually more homogeneous and tend to form much more uniform networks.

Methods for obtaining homogeneous and controlled nanowire arrangements have attracted increasing interest. Oxide nanotemplates such as anodic alumina have been used to separate and order metallic nanowires [77, 78]. Polydimethylsiloxane stamps have also been successfully used for dry transfer printing [11, 28, 79]. A wealth of other different and original techniques such as blown-bubble film technique [80], controlled dip coating [81, 82], microfluidic assembly [83, 84], electronic/magnetic field-assisted assembly [85, 86], convective force driven assembly [87-90], layer-by-layer assembly of nanowires [91-93], direct gravure printing [94], and electrospinning [37, 95] have also been attempted to produce high-quality ordered nanowire arrays.

One of the most important points to be addressed for device integration is the surface roughness and the contact resistance between nanowires. The former can be addressed by lamination [96] preventing shunts from occurring [52], embedding nanowires within a transparent polymer using a wet chemical process as demonstrated by Zeng et al [97] or by sandwiching a metallic nanowire network in between two $\mathrm{ZnO}$ layers [98]. Junction resistances can be lowered by annealing which can be made very spatially selective using plasmonic welding [32].

Lastly, improving thermal and more generally environmental stability is a critical issue to address for integrating nanostructures in devices. Embedding within oxide materials is proving to be a very promising way to achieve this. Indeed, it has been used to prevent the thermal degradation of silver nanowire networks [77, 95, 98-100]. Ramasamy et al [100] have shown that their $\mathrm{TiO}_{2}$ encapsulated silver nanowires are thermally stable up to a temperature of $750{ }^{\circ} \mathrm{C}$. Recently, Chung et al [73] and Kim et al [98] have demonstrated that such embedding is also beneficial for lowering junction resistances and smoothing the surface morphology, improving the mechanical adhesion to the substrate while maintaining good mechanical flexibility.

\section{Properties of TCM based on silver nanowire networks}

\subsection{Electrical properties}

The electrical properties of nanowire networks are dependent upon several characteristics of the nanowires themselves and of course the network morphology. In general the resistance of the network increases as the length of the nanowires used decreases, and this trend is continued if the wire diameter is decreased [35, 101]. This increase of resistance with decreasing wire diameter is due to an increase of the individual nanowire resistance. This phenomenon was studied by Bid et al [102] who attribute the increase in resistance to surface scattering of the electrons, as the diameter of the nanowire reduces close to or below the mean free path of electrons of the bulk material. In such case the role of surface scattering becomes dominant and causes a reduction of the mean free path then leading to an increase in the resistivity above that of the bulk.

The role of the nanowire length is less straightforward as the dependence is a direct result of the percolative nature of the conduction mechanism in nanowire networks. Hecht et al [103] take an elegantly simple view of the conduction mechanism to explain the dependence of network resistance on length - although initially considered for carbon nanotube networks it is also applicable for metallic networks. Put simply, the concept is that for a given network size the minimum number of nanowires required to cross it is inversely proportional to the length of the nanowires. The orientations of the nanowires determine the number of interconnections required to make the network percolate, but the minimum number is determined by the length of the wire. In this model the longer nanowires require fewer wires to make a conduction path across a given space, thus they have fewer junctions and fewer transfers between wires. This results in 

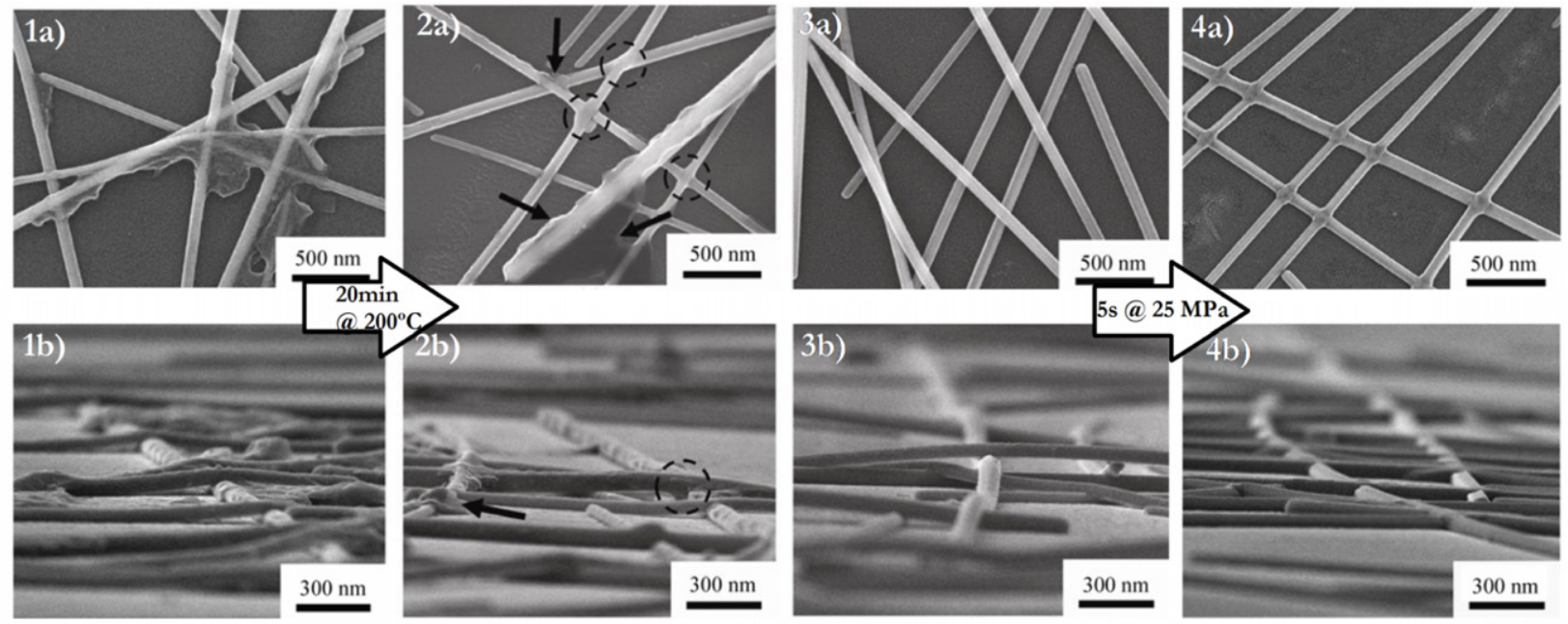

Figure 3. SEM images of AgNW networks, the (a) series are top-down views of the sample and the (b) series show off-angle cross-sectional views. Images 1(a) and (b) are of as-deposited nanowire networks. 2(a) and (b) show the effect of thermal annealing at $200{ }^{\circ} \mathrm{C}$ for $20 \mathrm{~min} .3$ (a) and (b) show an as-deposited network after rinsing in water and ethanol and 4(a) and (b) show the effect of $5 \mathrm{~s}$ of mechanical pressure applied at $25 \mathrm{MPa}$. Images adapted from [31] with kind permission from Springer Science + Business Media.

the lower resistances observed in networks made of long wires.

Beyond the dimensions of the wires themselves, the wire density plays a large role in the conduction properties of the network. Many studies have shown that increasing the number of wires decreases the resistance, however this also results in a decrease in the optical transmission of the network [11, 35, 37, 52, 97]. Striking a balance between the electrical and optical properties has long been the challenge for transparent conductive materials (see section 4.3). Among others, there are two specific advantages of nanowire networks as transparent conductive materials. One is the low sheet resistances achievable with nanowire systems which can be on the order of a ten ohms per square, and the other is the transparency in the infra-red region of the spectrum. This point will be covered in more detail in section 4.2.

The overall resistance of metallic nanowire networks depends on the material used to make the network, so far mainly copper and silver networks are being studied with the silver networks having the better electrical properties. Resistance of the network is also determined by the nanowire density and the junction resistance. The diameter and length of the nanowires play a crucial role in the required density for percolation and hence the number of conduction pathways through the network. Increasing the density of nanowires above the percolation threshold results in conduction pathways through the network that are in parallel, hence as the number of conduction paths increase the resistance decreases. The junction resistance is determined by the nature of the junction, this is dependent on how the nanowires are grown, purified, and deposited. Modification of the junction resistance has been studied in the literature and methods such as thermal annealing [11, 31, 59, 97] high pressure [31], electrical annealing [59], optical sintering [32] and encapsulation $[75,97]$ have all been shown to reduce the resistance of the network. Some examples of post-treatments and their effects on the morphology of the network are provided in figure 3 .

The $I-V$ characteristics of metallic nanowires at low voltages have been shown to be close to linear [32, 104] and at higher voltages the electrical breakdown mechanism of individual single crystal nanowires has been determined to be due to electromigration of atoms within the wires [104]. Although the nanowires will experience Joule heating as a result of current flow, the electrical resistance of the individual wires is low and the thermal conductivity of the wires is high, resulting in speedy distribution of thermal energy throughout the network. In such case it has been shown that electromigration will cause failure of the networks before the critical temperature to cause thermal failure is reached, as shown in figure 4.

The exception to this is the case in which the junction resistance is high-in such case the Joule heating localized at the junctions between nanowires in the network can act to increase the local temperature. Fortunately, this typically results in a reduction of the junction resistance through thermal sintering of the junction, which then reduces the heat produced at that point.

\subsection{Optical properties}

In this section, we focus on optically coupled metallic nanoparticle networks used as transparent conducting materials. Emphasis is given to cylindrical geometries.

Nanoscale particles are not new-neither in nature nor in science. Our ancestors put them to good use when fabricating magnificent colour pigments and glasses. The vivid colour of metal colloids, the blueness of the sky, all these observations are accounted for by elastic light scattering. When an incident electromagnetic field impacts on a metallic nanoparticle, the 


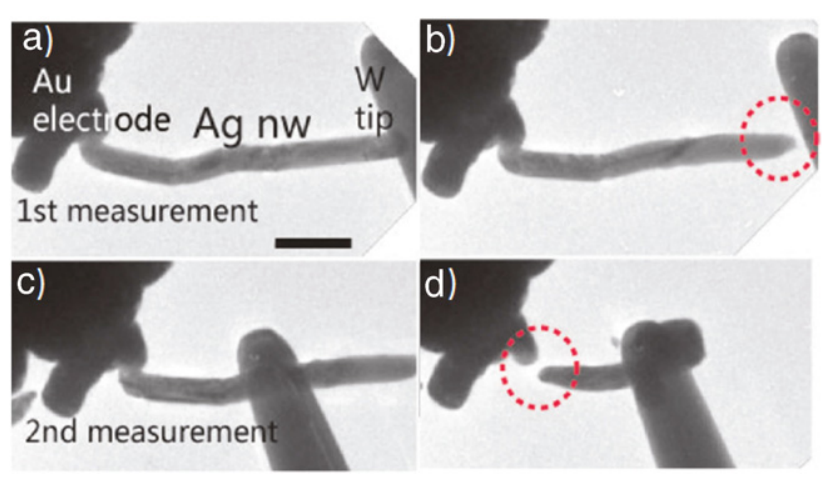

Figure 4. Electrical breakdown of a silver nanowire, two measurements were performed ( $\mathrm{ab}$ and $\mathrm{cd}$ ). The images show the wire before and after breakdown. Due to the position of the failure in each case it was determined that electromigration is the most likely candidate as the failure mechanism. Scale bar in (a) is $200 \mathrm{~nm}$. Reprinted with permission from [104], copyright 2013 American Chemical Society.

electric field component will set into collective motion both the bound and the free electrons with respect to fixed positive ions of the metal. This eventually leads to electronic (which are essentially independent of the particle size) and geometric resonances over some spectral regions, effectively polarizing the material under investigation. Such polarization occurs if the particle size (the radius $r$ of a spherical particle for example) is a multiple of the incident wavelength value. In that case, very narrow peaks are added to the extinction and scattering efficiencies. The former can be accurately described using the classical Drude-Lorentz-Sommerfeld equation for the resulting dielectric function:

$$
\varepsilon(\omega)=1-\frac{\omega_{\mathrm{p}}^{2}}{\omega^{2}+\mathrm{i} \omega \gamma_{\mathrm{fe}}}+\sum_{j} \frac{S_{j} \omega_{j}^{2}}{\omega_{j}^{2}-\omega^{2}-\mathrm{i} \omega \gamma_{j}}
$$

where $\omega_{j}$ and $\gamma_{j}$ are the resonant frequency and the damping constant of the $j$ th harmonic oscillator, respectively. $\gamma_{\mathrm{fe}}$ is the damping constant of the free electrons and $\omega_{\mathrm{p}}$ is the bulk plasma frequency [105].

In metal though, the contribution of free electrons dominates the dielectric function resulting in a remarkable effect of light coupling to metallic nanoparticles via the excitation of surface-plasmon polaritons (SPPs) (as opposed to 'bulk' plasmons) as theorized by the pioneering work of Ritchie [106] in the 1950s. Surface plasmons are simply plasmons confined to the surface and are directly responsible for the geometry dependence of nanoparticle optical responses. They have both longitudinal and transverse components and can actually be observed at high spatial resolution with electron-energy loss spectroscopy experiments [107, 108] or scanning near-field optical microscopy [109, 110]. Surface plasmons confined to a nanostructure are also called localized surface plasmons (LSPs).

SPPs stem from the relative difference of permittivities between the metals and the surrounding non-conducting (dielectric) media. For instance, for metallic cylinders of infinite length and small diameter, the resonance condition for

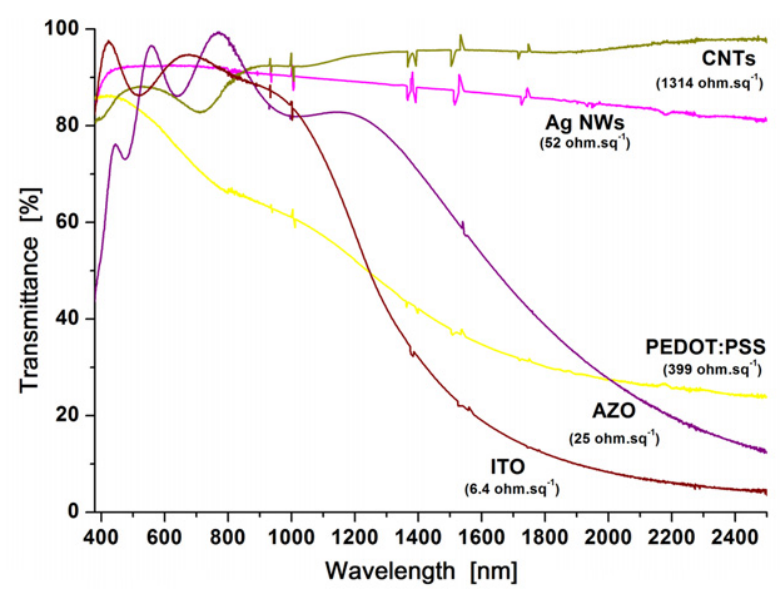

Figure 5. Vis-NIR optical transmittance spectra (substrate subtracted) of indium tin oxide, aluminium-doped zinc oxide, PEDOT:PSS, silver nanowires and single walled carbon nanotubes. The associated sheet resistance values are given in brackets.

an SPP resonance is given by $\varepsilon_{1}=-\varepsilon_{\mathrm{M}}$ and $\varepsilon_{2} \sim 0$ where $\varepsilon_{1}$ is the real part of the dielectric function of the particle, $\varepsilon_{\mathrm{M}}$ the dielectric function of the surrounding medium and $\varepsilon_{2}$ the imaginary part of the dielectric function $\varepsilon$ [105]. Surface plasmons can be damped by surface roughness, domain boundaries or any other sort of defect.

Having light being concentrated and channelled over the surface of nanoparticles as well as in between them results in a strong electromagnetic field enhancement. Indeed, the amplitude of the electromagnetic field observed in the vicinity of nanoparticles $[111,112]$ can amount to several hundred times that of the incident field in places called 'hot spots'. Garnett et al [32] put this effect to a practical use by performing plasmonic welding to assist the sintering of metallic nanowires into large interconnected networks. Spechler and Arnold also used this method but employed a pulsed laser to minimize the excess energy input [113]. This feature is also used for surface-enhanced Raman spectroscopy (SERS) [77]. These phenomena illustrate what is called dependent scattering or 'optical antenna effects'. Indeed, while individual nanoparticles exhibit a single or a few plasmon resonances, much more complex resonance spectra are observed for interacting and coupled structures. Figure 5 shows different optical transmittance spectra of transparent conductors in the visible/near-IR range.

The plasma frequency of indium tin oxide and aluminium-doped zinc oxide falls in the near-IR so that the optical transmittance drastically decreases in this region. AgNWs and carbon nanotubes do not show such a feature and demonstrate similar optical transmittance in the visible range. For applications requiring conductive films that are transparent in the IR region of the spectrum, nanostructured films of metallic nanowires or carbon nanotubes constitute therefore an excellent choice. In the case of AgNW networks, the transmittance loss is mainly accounted for by reflective scattering of light by the nanowires themselves [73]. In addition, there is a significant reduction of the reflectance since there is a refraction index gradient (directly resulting 
from having nanowires suspended in air) which is an inherent factor enabling better coupling of light to the material [114].

On a final note, the ratio of diffuse transmission to direct transmission of light (the haze factor) of a material can be considered a critical factor for some applications. As already briefly discussed in the introduction, the proportion of scattered transmitted light is an important parameter which should be considered alongside optical transparency and sheet resistance. The haze factor is associated with the degree to which transmitted light is scattered. It is strongly dependent on the experimental conditions and methods used for fabrication of the network. It is possible to fabricate a broad range of metallic nanowire networks with haze factors from $<2 \%$ to more than $30 \%$ [33]. This range allows metallic nanowire electrodes to be fabricated to suit many different applications. More specifically, a low haze factor is required for display applications with typical values below $2 \%$. Higher values are desirable for enhancing the path length of light in the absorbing layer of a solar cell, thus improving the photovoltaic efficiency [115]. Haze factor is usually not considered, for instance when calculating the FoM, however recent studies have justly focused on its importance [33, 116, 117]. Unfortunately it is still difficult to provide a fair comparison and discussion on AgNW-based electrode haze factor since the literature reports very dispersed data. Actually haze factor depends upon several parameters including NW density or diameter [33], deposition method and post-processing. Clearly, more work should be dedicated to this topic as it is of crucial importance for the optimal integration of $\mathrm{AgNW}$-based transparent electrodes in real devices.

\subsection{Optimizing the electro-optical properties}

Optimizing the properties of a TCM is a delicate challenge which depends upon the considered application. Such a task is associated with the inherent trade-off between the two requirements of electrical conductivity and optical transmittance. Material transparency can be improved by decreasing the film thickness for a thin film (such as TCO) or the density of conducting nanoparticles (such as AgNWs or CNTs or disordered arrays of exfoliated graphene flakes). However, a very low thickness or low density will result in significantly less conductive pathways available. This feature is illustrated in figure 6(a) where optical transmittance versus sheet resistance is plotted for graphene, single-wall carbon nanotubes (SWNTs), Cu and Ag NWs [118]. Such a set of data points is associated with a series of specimens with varying thickness or density. The data can be fitted by using two different regimes which can be ascribed to bulk-like and percolation behaviour [118]. For each material considered, the bottom-left portion of the data (fitted by the solid line and corresponding to thicker films) is associated with bulk-like materials for which dc conductivity is invariant with sample thickness. Another dependency should be considered for the high-transparency regime where deviation from bulk-like behaviour is observed and can be explained by percolation effects as revealed by the good fit between the dashed lines
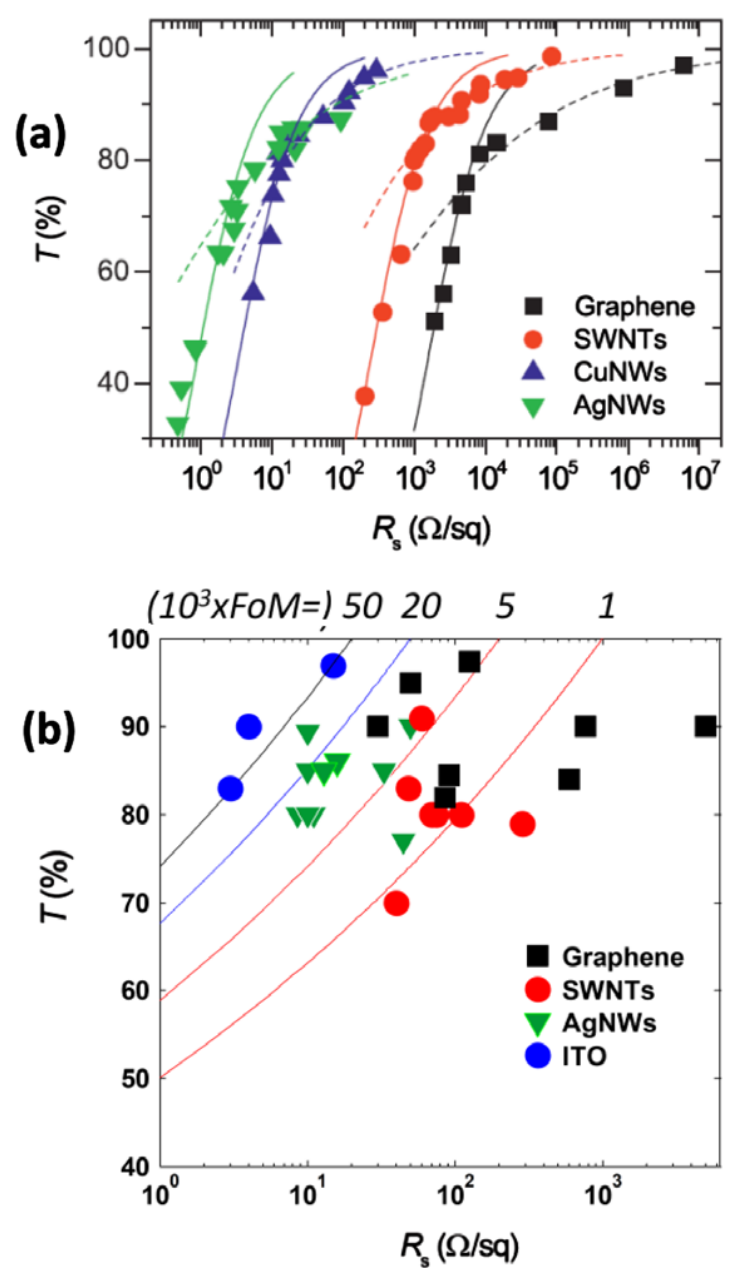

Figure 6. (a) Optical transmittance (at $550 \mathrm{~nm}$ ) versus sheet resistance for transparent conductors composed of nanostructured materials. Each set of points can be divided into two regimes: the bulk regime (solid line) and the percolation regime (dashed line). Reprinted with permission from [118]. (b) Optical transmittance (at $550 \mathrm{~nm}$ ) versus sheet resistance for graphene [14-19], carbon nanotubes [11, 20-26], AgNWs [27-33] and ITO [9, 11, 13]. Solid lines exhibit iso-values of the figure of merit (see text for explanations).

and the data in the top-right region of figure 6(a). When the density of nanowires or nanotubes is decreased the sample state approaches the threshold for network percolation. At this point the probability that the network will conduct is $50 \%$, as the percolation threshold across the network is less and less likely to contain a spanning conduction pathway and consequently the sheet resistance soars.

The balance between electrical and optical properties should be quantified to allow comparison of the performance of TCMs. Therefore figures of merit (FoMs) are considered based on the physical properties being pursued. The classical FoM definition of Haacke [119] is commonly used [9, 17];

$$
\mathrm{FoM}=\frac{T^{10}}{R_{\mathrm{S}}}
$$

where $T$ is the optical transmittance measured at a wavelength of $550 \mathrm{~nm}$, which corresponds to the maximum human visual 
sensitivity and the peak in photon output of the Sun. It is noteworthy that another FoM often considered is the ratio between the electrical conductivity and the optical absorption coefficient.

Figure 6(b) exhibits experimental optical transmission and sheet resistance data associated with graphene [14-19], CNTs [11, 20-26], Ag NWs [27-33] and ITO [9, 11, 13]. The lines show iso-values of the FoM defined by equation (2). The larger the FoM values, the better the associated electro-optical properties (so the ideal region corresponds to the top-left of this graph). While a dispersion of the data points can be observed, general trends can be stated. ITO properties are still the best, while metallic nanowires exhibit rather promising ones. CNTs are associated with lower FoM values because of the high junction resistance between nanotubes. Graphene exhibits rather scattered data and except for some studies, such as by Bae et al [18], suffers from rather large values of sheet resistance which still excludes it from applications like solar cells where requirements are the most stringent in terms of FoM.

\subsection{Thermal properties}

The thermal properties of metallic nanowires concern their thermal conductivity, the temperature dependence of their electrical conductivity as well as their thermal stability.

In recent years, numerous studies of the electrical transport properties of nanostructures have been reported thanks to the possible applications in miniaturized devices [120]. The mainstream research activities in this field concern oxide or semiconductor nanowires. Few investigations have been reported so far on the thermal conductivity of metallic NWs when compared to electrical conductivity. This stems from the fact that thermal and electrical conductivities of metals are intimately related through the Wiedemann-Franz relation, and electrical conductivity is generally directly accessible from an experimental view point. However, recently, the thermal conductivity $(K)$ of $\mathrm{Pt}$ nanowires (diameter of $100 \mathrm{~nm}$, length of $10 \mu \mathrm{m}$ ) was compared with measurements of electrical conductivity $(\sigma)$ at room temperature. Compared with bulk data, electrical and thermal conductivity of the nanowire are decreased by a factor of 2.5 and 3.4, Consequently, the Lorentz number $L=K /(\sigma T)$ of the nanowire was found to be smaller when compared with the bulk Lorentz number of metals, the difference being $25 \%$ [121]. This decrease is often related to the grain boundary scattering of electrons. Indeed, usually small-diameter NWs exhibit larger density of extended defects (such as grain boundaries) which affect the electrical resistivity. Other investigations found electrical properties of Au NWs similar to that of bulk without evidence of any polycrystalline grain structure [122] even with a diameter of $55 \mathrm{~nm}$.

When used within an organic material, an AgNW network can substantially increase the thermal conductivity of the organic matrix [123]. AgNW array-polymer composites have also been investigated as thermal interface material for use as a heat sink in electronic cooling applications [124]. Moreover, AgNW networks can also be used for the fabrication of flexible transparent thin-film heaters. Thanks to the intrinsic conductive properties of random AgNW networks above the percolation threshold, it is possible to combine flexibility, transparency and high heating performance at low voltage (i.e. $12 \mathrm{~V}$ ) which is of interest for many applications like defrosting, defogging or fabrication of thermo chromic displays [59].

The electrical resistivity $\rho$ of metallic nanowires themselves is a topic of current interest as well, since this is likely to be the dimension of metallic interconnections in future electronic devices. In the regime where the metallic NWs have a diameter of a few tens of nanometres $\rho$ depends upon the metal itself through its defects (impurities, extended defects such as grain-boundaries or twin boundaries) and their size [102]. Indeed, the electrical resistance for $\mathrm{Ag}$ and $\mathrm{Cu}$ of diameters ranging from 15 to $200 \mathrm{~nm}$ adhere well to the Bloch-Gruneisen model [102] showing only slight differences with bulk metals. The resistivity of the wires increases when the wire diameter $\left(D_{\mathrm{NW}}\right)$ decreases, especially when $D_{\mathrm{NW}}$ is comparable with the mean free path of the electrons (which is about $50 \mathrm{~nm}$ for bulk Ag [125]). This is due to the dominance of surface scattering effects [126] but also because low-diameter NWs often exhibit larger defect densities that induce electron scattering.

Few thorough investigations of the electrical contacts between two metallic NWs have been reported yet. However, they seem to play a crucial role as one can see from the electrical resistance evolution of $\mathrm{AgNW}$ networks during thermal annealing. Indeed, thermal annealing is an effective method to form fused contacts between NWs which drastically reduces their electrical resistance. The occurrence of local sintering is the driving force of such an evolution of the electrical properties.

Such thermal annealing exhibits a thermal limit above which the metallic NWs become unstable. This is a case of classical interfacial instability when dealing with liquid or solid cylinders when heated, called the Plateau-Rayleigh instability [127]. The morphological evolution of metallic NWs have been intensively investigated recently for $\mathrm{Au}$ [128], $\mathrm{Cu}$ [129], Pt [130], Sn [131], or Ag [132]. In a simple approach, perturbations with wavelengths larger than the original cylinder diameter become unstable. Observations show that upon thermal annealing metallic wires undergo various configuration changes. These lead to the wires breaking into chains of nanospheres at a much lower temperature than the melting point of the metal due to this capillary instability. Obviously, such thermal instability is very detrimental to the electrical conduction of the NW network. Therefore thermal annealing under controlled conditions should be carefully considered for optimizing electrical properties of the metallic NW network. The critical temperature at which this instability occurs is dependent on the nature of the nanowires, specifically the diameter and crystallographic structure. The material used also plays a role through its intrinsic properties (surface tension, diffusion coefficients, etc). 
A solution for enhancing thermal stability towards higher temperature is to embed the NWs within another material, for instance an oxide. A recent investigation shows indeed that AgNWs can be efficiently coated with uniform $\mathrm{TiO}_{2}$ shells [100]. In situ TEM thermal annealing observations showed that the AgNWs core remains intact until temperatures of $750^{\circ} \mathrm{C}$ [100]. Due to this improvement of thermal stability, such coated metallic nanowires can be heated sufficiently to be used as a reusable surface for SERS [77].

\subsection{Structural and mechanical properties}

The morphology of metallic nanowire networks depends greatly on the fabrication method employed for the network production. However, the majority of the current processes result in a rough surface morphology that may have a detrimental impact on the operation of devices. Out-of-planeoriented wires result in an electrode geometry that is not strictly planar. This leads to leakage currents, short circuits and recombination sites in photovoltaic systems. Several methods have been shown to reduce the problems associated with stray nanowires. High mechanical pressure has been shown to increase uniformity in the network geometry and reduce the $3 \mathrm{D}$ morphology to a true $2 \mathrm{D}$ network [31]. Encapsulation in transparent conductive polymers has also been shown to significantly reduce surface roughness [96, 133].

Understanding the structural behaviour of such networks has proven to be an interesting field of research and one of the main advantages of nanowire-based electrodes arises from the mechanical properties of nanowire networks. Recognizing that these electrodes are made of metals, it is no surprise that they show excellent properties when submitted to mechanical stress (either compression, tension or torsion). All have little to no effect on the electrical resistance of electrodes manufactured with metallic nanowires [11, 97, 134]. Figure 7 shows a comparison of three transparent conductive materials under repeated bending to a radius of curvature of $5 \mathrm{~mm}$. The ITO films used for this experiment were commercially purchased ITO on PET from Sigma Aldrich, initial sheet resistance was $\approx 60 \Omega / \square$ and film thickness is $\approx 300 \mathrm{~nm}$. FTO films were deposited by spray pyrolysis as described by Consonni et al [135]. The FTO film had a thickness of $250 \mathrm{~nm}$ and sheet resistance of $\approx 10 \Omega / \square$. The AgNW networks were deposited by spin coating and had a surface density of approximately $100 \mathrm{mg} \mathrm{m}^{-2}$ and sheet resistance of $\approx 16 \Omega / \square$. Clearly, AgNW networks provide a significant advantage for flexible electronic applications. The reduced resistance $\left(R-R_{0}\right) / R_{0}$ depicted in figure 7 indicates the ratio of the change in resistance divided by the initial resistance.

It has been shown that solar cells, organic light-emitting diodes (OLEDs) and thermal heaters can be produced on flexible substrates with nanowire electrodes. The ability of these electrodes to withstand external mechanical stresses is far above that of most traditional transparent conductive material. Even crumpling of such transparent electrodes has been demonstrated recently after deposition of nanowires on

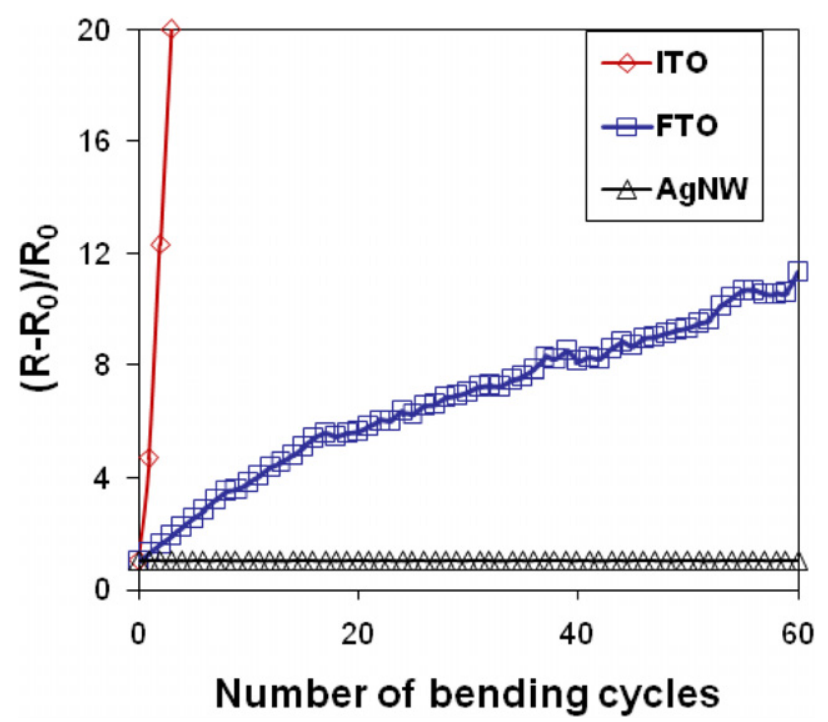

Figure 7. Comparison of TCMs under mechanical bending. During one cycle the specimen is flat, and then bent up to a radius of curvature of $5 \mathrm{~mm}$, and returned to the initial position. The TCM is on the opposite side to the bending radius and is therefore placed under tension.

a very thin $(1.3 \mu \mathrm{m})$ piece of polyethylenenaphtalate without changing the electrical properties [59].

The major weakness of metallic nanowire networks in the face of mechanical stress is adhesion of the layer to the substrate material. If friction or adhesive tape are applied to the network directly, low adhesion of the network to the substrate generally causes the network to fail. If, however, the network is placed under mechanical stress that is applied to the substrate of the network, it has an excellent ability to follow the substrate flexion. When the force is applied directly to the network in the form of rubbing or scraping the adhesion of the nanowires generally fails. This can be reduced by encapsulating the conductive network but the feasibility of such an encapsulation depends on the desired application.

Recently, a further exhibition of the remarkable mechanical properties of metallic nanowire materials has emerged with several papers on stretchable electronic devices using nanowire networks as the conducting pathways [136-138]. These materials utilize metallic nanowire networks that when placed under strain 'unfold' to expand and fill the space on the stretched surface of the substrate. The work by $\mathrm{Xu}$ et al [137] used short AgNWs to produce the networks-in order to enable the electrodes to maintain conductivity at high strain, networks with high nanowire densities were created. These networks were not transparent but provided a rare opportunity for stretchable electronics. Lee et al [136] carried this work further to enable transparent stretchable electrodes and managed to achieve networks with an optical transmission of approximately $90 \%$ and sheet resistance of between 9 and $70 \Omega / \square$ that was able to withstand over $460 \%$ strain without significant increase in electrical resistance. This result was achieved by using comparatively low densities of very long (200-500 $\mu \mathrm{m})$ AgNWs [136]. 


\section{Simulation appreaches of the physical properties of metallic nanowire networks}

\subsection{Simulation of electrical properties}

The electrical properties of nanowire networks can be simulated by the application of percolation theory to a $2 \mathrm{D}$ stick model [139-141]. These computational approaches to the conduction through nanowire networks allow exploration of the effects of variables such as nanowire length, diameter and density on the electrical properties of the networks. The Monte Carlo method [103, 139, 142] has been used to statistically simulate metallic nanowire networks and through the combination of topology and experimental data it is possible to model the electrical properties of a randomly oriented network. Much work has been done in the realm of percolation theory in relation to $2 \mathrm{D}$ conducting sticks [139-145] but in terms of directly applicable realworld modelling of these systems the field is still in its infancy. In relation to this modelling where simulations are generated, including experimentally determined values such as length distribution or junction resistance, some results are beginning to emerge. The work of Coleman et al [118, 146, 147] combined experimental and theoretical approaches to the problem of metallic nanowire network percolation. Combining AFM current mapping of networks with simulations and direct measurements of the nanowire junction resistances has enabled the establishment of a strong relationship between their experimental and theoretical work.

The weakness in the current literature is that almost universally the nanowires in the systems modelled are a widthless stick of unit length. This restriction is useful for rapid calculation but is an approximation that may lead to inaccurate simulations. Including length distributions in the simulation and exploring the effect of wire diameter on the occurrence of percolation is an important next step.

On-going modelling of nanowire networks will enable the prediction of size effects, optimization of optoelectronic properties and should lead to a set of selection criteria that will allow nanowire dimensions to be engineered for specific device applications.

Such numerical simulations should help to better define the optimal properties and to predict the optimal device geometry required to meet the needs of a given application. For instance, simulations can provide a starting point concerning the nanowire density or geometry which will result in a good compromise between optical transmission and electrical conduction. The latter can be tackled with the percolation theory while the simulations related to the optical properties are discussed below.

\subsection{Simulation of the optical properties}

Simulation of optical properties at the nanoscale is a very active field of research, for the reasons outlined previously. For instance, Lee et al [27] have shown that both the optical transmission and electrical conductance of periodic metal nano-gratings are in theory and experimentally greater than those of ITO. Since Maxwell's equations can be solved at the microscopic level, the computing methods presented here are all based on solving these first-principle equations. Historically, light scattering by single spherical nanoparticle was the first nanoscale property to be successfully simulated. This was achieved using the classical Mie scattering theory by providing an exact solution to Maxwell's equations [148]. Although Mie's work refers to light scattering from a spherical particle only, the discretized Mie formalism provides an appropriate framework to describe more complicated shapes [149] such as hexagonal cylinders [150] and even aggregates. These different methods based on Mie theory to solve more complex scattering problems are often termed generalized Mie theory (GMT).

However, when it comes to particles of arbitrary shape, semi-analytical or numerical methods must be used. The transfer matrix method (or null-field method) [151] which is a semi-analytical approach is very attractive for computing light scattering from complex structures. However, similarly to Mie theory, there are issues regarding the treatment of high-aspect-ratio nanoparticles such as cylinders [152, 153]. Nonetheless, Larciprete et al [154] recently used this method to derive the transmission and reflection coefficients of random-oriented AgNWs in the IR range. Numerous purely numerical methods have also emerged over the past few decades to solve Maxwell's equations in diverse confined geometries. They usually make it possible to simulate efficiently a broader range of particle geometries and arrangements than the GMT. To name a few, the discrete dipole approximation (DDA) [155-157] is useful to describe isolated nanoparticles while the finite difference time domain (FDTD) [158-160] method is very powerful in describing grid geometries (as well as arbitrary structures) along with the Fourier modal method (FMM) [161] which is related to rigorous coupled-wave analysis (RCWA) [162]. Relatively unused until recently, the finite element method (FEM) [27, 163, 164] is now becoming increasingly popular, especially for describing complex nanowire arrangements.

All these previously mentioned first-principle methods work very well with either a single, a very limited number of particles or ordered nanoparticle arrays. However, these approaches often break down when it comes to modelling the optical properties of random nanoparticle networks.

Using a statistical approach proves to be more adequate to the problem [165-167]. The random walk approach [166-168] correctly represents the random motion of photons through a three-dimensional structure, although the photon mean free path must remain small [169]. Moreover, in a collection of randomly oriented particles, the quantities of interest are the average scattering and absorption cross sections since the distinction between individual nanowires can no longer be made. Computing these average values has the advantage of taking into account de facto dependent scattering which would otherwise be conceptually and computationally very difficult to model. 


\section{Applications}

Due to their low sheet resistance and high transparency, AgNW-based electrodes are good candidates for device integration in existing applications. Their mechanical properties also provide opportunities for integration in emerging flexible applications. As has been shown previously, this technology is compatible with low cost and large-scale processes. However, some solutions to avoid several drawbacks have to be found to enable successful industrial integration into devices.

First of all, let us clarify that although the cost of the bulk silver used to fabricate nanowire films is sometimes considered as an issue for this emerging TCM, the extremely small amount of raw metal needed renders this option economically relevant. Therefore, the use of a noble metal is not itself an economic issue. For instance, a cost analysis of $\mathrm{PV}$ modules suggests that it is three times less expensive with AgNW compared to ITO on PET [34].

In terms of applications, control of metallic network roughness is also a major challenge [27] that has to be taken into account. Indeed, a major issue comes from the protruding nanowires from the network that can lead to electrical shortcuts or leakages in multilayered devices. Roughness of bare AgNW electrode has been evaluated to be in the order of hundreds of nm [52, 97] (RMS 30-1000 and 200-300 nm top-to-bottom height). In the literature, different strategies have been developed to circumvent electrode roughness. Surface roughness reduction has been achieved by mechanical pressing [52, 96], thermal annealing [27], polymer coating [96, 97] surface peeling and transfer processes $[96,97,133]$. It has been shown that the reduction of surface roughness by flattening the network not only reduces the risk of short circuit but also decreases sheet resistance.

As described earlier, a shortcoming of AgNW electrodes is the poor adhesion of the nanowire network to the substrate. This issue can be easily circumvented in device applications by encapsulation with a thin layer of organic materials (Teflon, PEDOT:PSS... ) $[11,52]$. Typically devices can be designed in such a way that the adhesion of the nanowire network to the substrate becomes less important as it is incorporated into a multilayered device.

Finally, an extended study of AgNW-based device ageing has not yet been reported. Nevertheless, it is certainly closely related to oxidation and sulfuration of nanowires as well as to electromigration [97]. Device ageing is not directly correlated to Ag NW electrode ageing and will be dependent largely on the overall architecture of the device and not solely on the AgNW layer. Indeed, due to multilayer stacking, it is a complex mechanism and further studies are needed to explain possible alteration of electrodes in the long term. However, flexible Ag nanowire electrodes appear rather stable; they keep their optical and electrical performances for more than a year in air, without special care.

In addition to the potential technological integration issues that have been previously listed, device optimization should also require tuning the work function of the electrodes [170] to ease charge carrier injection or collection in optoelectronic devices with any active layers. For this purpose, transparent flexible electrodes can for instance be modified by coating with another transparent layer (organic compounds, metallic oxides... ) [27, 171, 172].

\subsection{Solar cells}

Many groups are beginning to incorporate silver nanowires as front electrodes for solar applications [99, 134, 171, 173-176]. Depending on the experimental conditions used for their fabrication, metallic nanowire networks can have haze factors varying between 1 and 30\% [33]. If low haze factor is desired, this can be achieved by using spray deposition and spin coating which can lead to haze factors below $2 \%$. Such haziness is acceptable for display and window applications. Fabrication and transfer of nanowire networks by vacuum filtration has demonstrated nanowire networks exhibiting haze factor of approximately 30\% over the 400-900 nm spectral range [33]. For application in photovoltaic systems a high haze factor implies light scattering into the device and results in a larger effective absorption cross section. As demonstrated by Chih-Hung et al, increasing haze results in an increase in efficiency [115]. Gaynor et al [171] demonstrated approximately a $10 \%$ increase in conversion efficiency by using $\equiv$ nanowire composite electrode over an ITO standard $(\square=3.4 \%$ using ITO on plastic and $\square=3.8 \%$ for $\mathrm{Ag}$ nanowire composite). By using low-haze networks and taking advantage of the metal nanowire network transparency in the IR region of the spectrum, it is possible to create solar cells semi-transparent in the visible region [174]. These cells absorb some of the light in the visible region and much of the infra-red, resulting in an average transmission of about $61 \%$ in the $450-650 \mathrm{~nm}$ wavelength range [174]. This type of solar cell could be used to provide tinted windows that produce energy whilst reducing glare. An advantage of metallic nanowire networks is demonstrated in the production of flexible solar cells [173], which is unlikely to be achievable on any transparent conductive oxide. The flexible solar cells show a remarkable ability to produce electricity even when bent to angles of $120^{\circ}$, as demonstrated in figure 8 . These flexible solar cells are organic polymer cells that incorporate AgNW networks embedded in PEDOT:PSS, a conductive polymer.

\subsection{Transparent heaters}

Transparent heaters have a wide variety of uses. Defrosting windows in aeroplanes was one of the first applications, allowing flight at high altitude [10]. Most defrosting or defogging transparent devices are fabricated using layers of ITO. But as explained before, such material does not have significant resistance to mechanical constraints. Thus random networks of silver nanowires appear to be well adapted if one wants to make transparent film heaters on conformable or flexible surfaces. The operating principle is based on Joule's law. This rule, also known as Joule heating, describes the production of heat as the result of a current flowing through a conductor. When an electric current flows through a transparent conductor, electric energy is converted to heat 

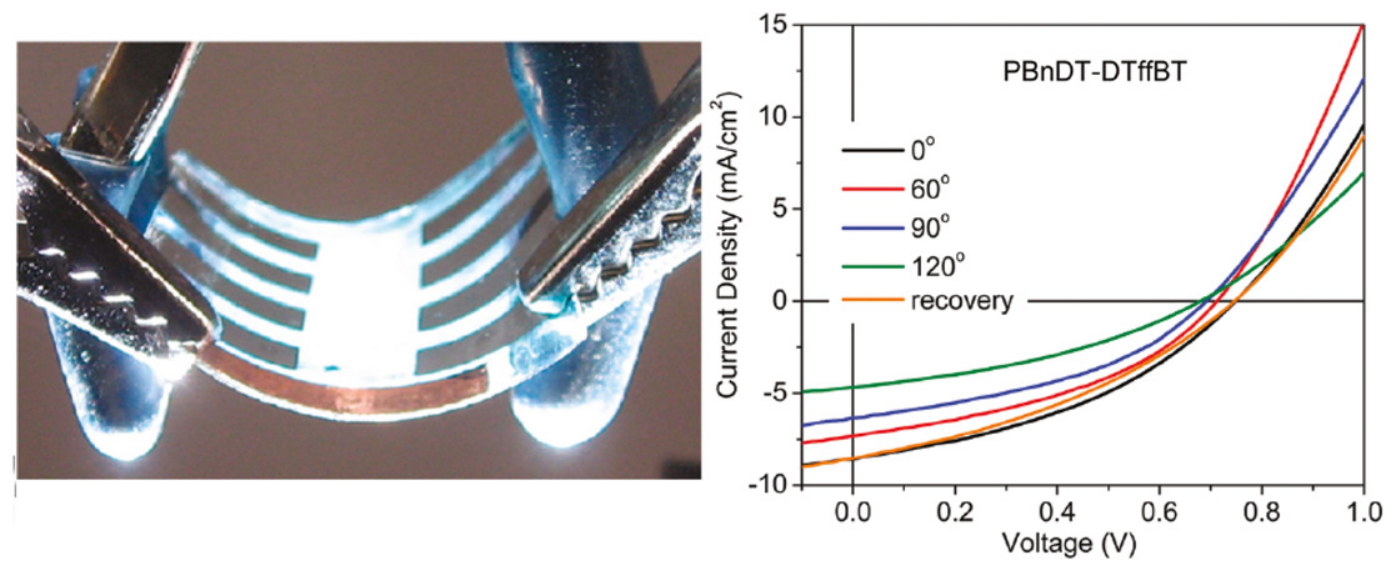

Figure 8. Flexible solar cell in operation (left) and the $I-V$ curves at different angles. Reprinted with permission from [173], copyright 2013 American Chemical Society.

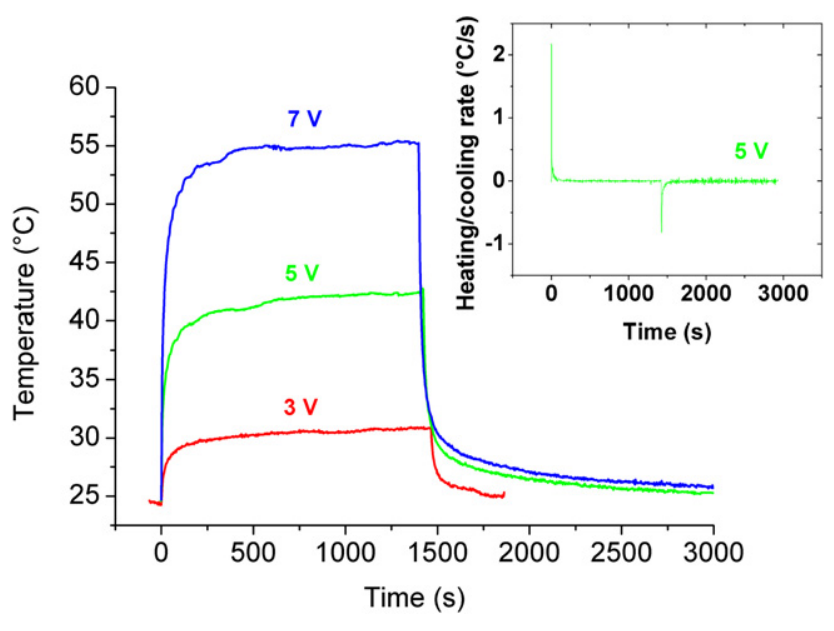

Figure 9. Heating experiments showing temperature as a function of time for a $35 \Omega / \square$ film heater on a $125 \mu \mathrm{m}$ PEN substrate at different applied voltages. Inset: derivative of the temperature versus time at $5 \mathrm{~V}$ applied bias. Reproduced with kind permission from Springer Science + Business Media [59].

through resistive losses in the material. It can be written according to the equation $P=V^{2} / R$, where $V$ is the applied voltage, $R$ the total resistance and $P$ the dissipated power.

As has already been explained, limitations of current in existing commercial technologies can be circumvented by the adequate use of nanomaterials. For this application, devices with excellent properties were reported recently with silver nanowires, in particular on plastic substrates (see figure 9) [59, 177, 178]. Interestingly, the excellent optoelectronic properties of $\mathrm{AgNW}$ random networks allow the delivery of significant power at low voltage (typically below $12 \mathrm{~V}$ ) which is more suitable for many applications.

\subsection{Displays (OLED)}

A key advantage of OLEDs or PLEDs (polymer light-emitting diodes) is that they can be made entirely by solution process and thus are more suitable for low-cost, large-area flexible displays and white lighting panels. OLED materials need current flowing through the active layer to light up. This means that charge carrier transport properties play an important role in such devices. Not only the properties of the active layer itself, but also the interfaces with their neighbouring layers and the electrodes are of overwhelming importance [179]. A few studies have examined metallic nanowire-based OLEDs: good electroluminescent properties have been reported $[97,133,170,180]$. In particular, Yu et al have integrated AgNW polymer composite electrodes in large angle bending PLEDs (see figure 10).

As in OPV devices, a slight increase of device performance through higher power efficiency of AgNW-based OLED devices compared to conventional ITO has been observed [27, 97, 170, 181-183]. This is partly ascribed to an enhanced scattering of emitted light by AgNWs. Recently, Gaynor et al have demonstrated very high-efficiency ITO-free white OLEDs [179]. The highest luminous efficacy for an ITO-free white OLED with stabilized viewing angle and almost perfect Lambertian emission was reported (see figure $10(d-1))(b)$. These first studies open the way towards large-scale integration of AgNW-based electrodes in emerging flexible display devices.

\subsection{Touch panels}

Touch-screen technology has become very popular in many electronic products. It is widely used for smartphones, tablet $\mathrm{PCs}$, game consoles, electronic information, ticketing kiosks and other technologies. Few approaches are available, but the main industrial products currently used are based on resistive or capacitive touch panels. Since emitted light from the device comes through the front electrode, it requires a high transparency with low diffusivity (low haze factor). It is very likely that in forthcoming years many displays (in particular for smartphones) will become flexible, and consequently new bendable transparent conductors will be mandatory for these applications. The AgNW-based electrodes appear to be promising candidates, as recently reported using resistive-type panels [79, 184]. Some of the authors of this review also fabricated capacitive touch sensors [69], demonstrating 

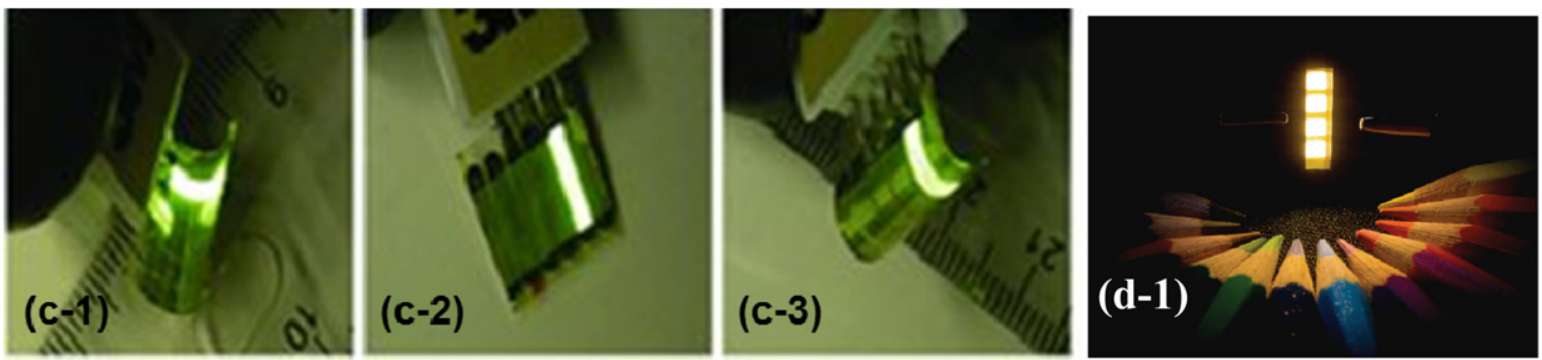

Figure 10. Photographs of shape memory PLEDs emitting at around $300 \mathrm{~cd} \mathrm{~m}^{-2}$, bent to a $2.5 \mathrm{~mm}$ radius convex (c-1), recovered to flat shape after heating at $120^{\circ} \mathrm{C}$ for $1 \mathrm{~min}(\mathrm{c}-2)$, and bent in the opposite direction as in (c-1) to a $2.5 \mathrm{~mm}$ concave radius (c-3). Photographic image of four operating white tandem OLEDs at $1000 \mathrm{~cd} \mathrm{~m}^{-2}$ (d-1). Reprinted with permission from [133, 181], copyright $2013 \mathrm{Wiley}$.

a)
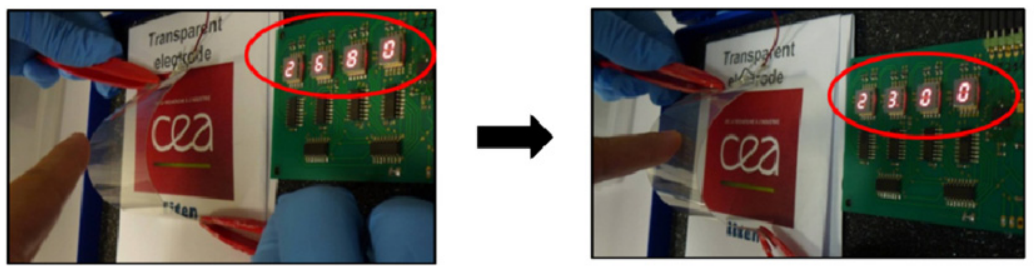

b)

c)
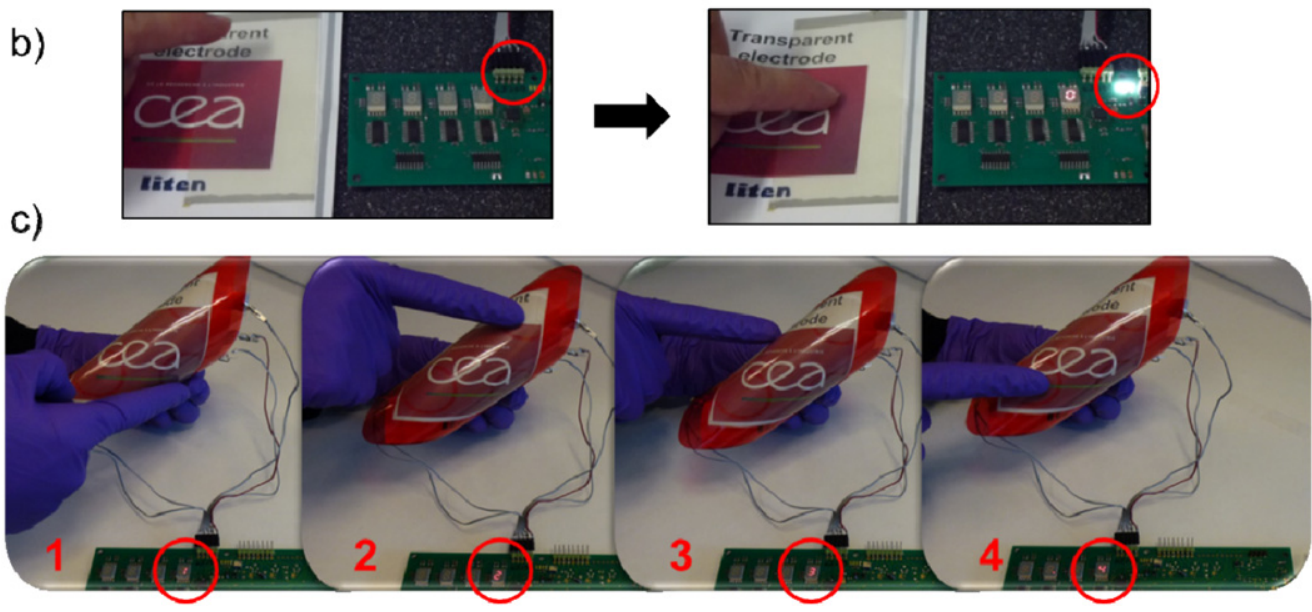

Figure 11. (a) Left, the finger does not touch the sensor surface and oscillation value is 268 . Right, the finger touches the surface and oscillation value is 230. (b) Left, finger is far from the surface thus the LED is off. Right, the finger is touching the sensor which switches on the LED. (c) Recognition of each electrode (1-4) [69].

that AgNW-based technology offers good performance for different possible technologies and has the potential to replace in the short term ITO for future flexible touch-screen panels (see figure 11). The strength of nanowire networks over ITO or other oxides in the field of flexible electronics lies in the fact that the oxide family is typically brittle resulting in short lifespans of structures deposited on flexible materials.

It was also recently demonstrated that flexible transparent capacitive sensors can be used for the detection of deformation and pressure. Capacitive sensor sheets fabricated by employing AgNW networks embedded in the surface layer of a polyurethane matrix exhibit good stretchability and transparency [185].

\section{Closing remarks and future work}

We have shown that Ag NW networks offer interesting opportunities for fundamental as well as applied research.
Thanks to the ease to fabricate and network, and also to the excellent electrical, optical and thermal properties of silver, AgNW networks exhibit great potential for use as flexible transparent conductor in several applications including flat screens, solar cells and transparent heaters. We have discussed the fabrication of AgNWs and their main properties. The structural arrangement of the network as well its physical properties (optical, electrical or mechanical) and its modelling (using percolation models) have started to be investigated lately with increasing effort to improve these properties. Thermal or chemical stability are often mentioned as the main potential drawback of these nanostructures. However, further studies are needed to determine the real scope and limitations, and several post-treatments like coating and annealing have already proven useful in solving some of these limitations. The large range of haze factors achievable with AgNW networks (1-30\%) provides a material that can exhibit advantages in solar cell applications but is still 
suitable for displays and windows. This, combined with their mechanical flexibility, opens a broad application range. Several investigations have shown that the use of AgNW networks can enable new technologies such as flexible solar cells, flexible heaters, flexible touch-screens and stretchable electronics. In light of the successful application of this emerging TCM to these fields, metallic NW networks appear to be a key contender in emerging nanomaterials.

Further investigations related to this nanostructured material should be devoted to more precisely understand and optimize the deposition and post-treatment processes (thermal annealing, mechanical pressing, plasmonic welding, encapsulations, etc) needed to reduce sheet resistance and increase optical transmission as well as haze factor tuning. Also, the roughness associated with such networks should be considered to allow better integration in the various (often multilayered) devices. The optical simulations of important plasmonic effects in nanostructured random networks and the local percolation aspects (ensuring for instance an efficient collection of the generated carriers) should also be addressed in the future. The association of metallic NW networks with other very thin films such as graphene or carbon nanotubes could also become a new emerging nanomaterial with potentially very interesting properties.

Though this is a recent technology, it offers extremely promising results that should be confirmed in the next few years. Both nanoscience and industrial applications should benefit from the development of this innovative flexible transparent conductive material.

\section{Acknowledgments}

We thank Y Bréchet, A Carella, J Garnier, C Jimenez, D Nguyen and D Shultz for fruitful discussions. This work has been supported by the French Research National Agency (ANR) through the Habitat Intelligent et solaire photovoltaïque program (project ASYSCOL no. ANR-08HABISOL-002), by the Carnot Energies du Futur under the Project ET Nano (contract No. 10B792020A). PhD grants supporting this work were provided by Grenoble INP through a SEI programme, the DGA (French Ministry of Defence) and the European Union Erasmus Mundus programme through the IDS-FunMats (International Doctoral School of Functional Materials).

\section{References}

[1] Kumar A and Zhou C 2010 The race to replace tin-doped indium oxide: which material will win? ACS Nano 4 11-4

[2] Hu M, Gao J, Dong Y, Li K, Shan G, Yang S and Li R K-Y 2012 Flexible transparent PES/silver nanowires/PET sandwich-structured film for high-efficiency electromagnetic interference shielding Langmuir 28 7101-6

[3] Yu Y-H, Ma C-C M, Teng C-C, Huang Y-L, Lee S-H, Wang I and Wei M-H 2012 Electrical, morphological, and electromagnetic interference shielding properties of silver nanowires and nanoparticles conductive composites Mater. Chem. Phys. 136 334-40
[4] Rai T, Dantes P, Bahreyni B and Kim W S 2013 A stretchable RF antenna with silver nanowires IEEE Electron Device Lett. 34 544-6

[5] Komoda N, Nogi M, Suganuma K, Kohno K, Akiyama Y and Otsuka K 2012 Printed silver nanowire antennas with low signal loss at high-frequency radio Nanoscale 4 3148-53

[6] Yang C, Gu H, Lin W, Yuen M M, Wong C P, Xiong M and Gao B 2011 Silver nanowires: from scalable synthesis to recyclable foldable electronics Adv. Mater. 23 3052-6

[7] Hecht D S, Hu L and Irvin G 2011 Emerging transparent electrodes based on thin films of carbon nanotubes, graphene, and metallic nanostructures Adv. Mater. 23 1482-513

[8] Hu L, Wu H and Cui Y 2011 Metal nanogrids, nanowires, and nanofibers for transparent electrodes MRS Bull. $36760-5$

[9] Ellmer K 2012 Past achievements and future challenges in the development of optically transparent electrodes Nature Photon. 6 809-17

[10] Gordon R G 2000 Criteria for choosing transparent conductors MRS Bull. 25 52-7

[11] De S, Higgins T M, Lyons P E, Doherty E M, Nirmalraj P N, Blau W J, Boland J J and Coleman J N 2009 Silver nanowire networks as flexible, transparent, conducting films: extremely high DC to optical conductivity ratios ACS Nano 3 1767-74

[12] Chopra K L, Major S and Pandya D K 1983 Transparent conductors-a status review Thin Solid Films 102 1-46

[13] Sierros K A, Cairns D R, Abell J S and Kukureka S N 2010 Pulsed laser deposition of indium tin oxide films on flexible polyethylene naphthalate display substrates at room temperature Thin Solid Films 518 2623-7

[14] Blake P et al 2008 Graphene-based liquid crystal device Nano Lett. 8 1704-8

[15] Eda G, Fanchini G and Chhowalla M 2008 Large-area ultrathin films of reduced graphene oxide as a transparent and flexible electronic material Nature Nanotechnol. $3270-4$

[16] Kim U et al 2013 A transparent and stretchable graphene-based actuator for tactile display Nanotechnology 24145501

[17] Barnes T M, Reese M O, Bergeson J D, Larsen B A, Blackburn J L, Beard M C, Bult J and van de Lagemaat J 2012 Comparing the fundamental physics and device performance of transparent, conductive nanostructured networks with conventional transparent conducting oxides Adv. Energy Mater. 2 353-60

[18] Bae S et al 2010 Roll-to-roll production of 30-inch graphene films for transparent electrodes Nature Nanotechnol. 5 574-8

[19] Reina A, Jia X, Ho J, Nezich D, Son H, Bulovic V, Dresselhaus M S and Kong J 2009 Large area, few-layer graphene films on arbitrary substrates by chemical vapor deposition Nano Lett. 9 30-5

[20] Nirmalraj P N, Lyons P E, De S, Coleman J N and Boland J J 2009 Electrical connectivity in single-walled carbon nanotube networks Nano Lett. 9 3890-5

[21] Doherty E M, De S, Lyons P E, Shmeliov A, Nirmalraj P N, Scardaci V, Joimel J, Blau W J, Boland J J and Coleman J N 2009 The spatial uniformity and electromechanical stability of transparent, conductive films of single walled nanotubes Carbon 47 2466-73

[22] Tenent R C, Barnes T M, Bergeson J D, Ferguson A J, To B, Gedvilas L M, Heben M J and Blackburn J L 2009 Ultrasmooth, large-area, high-uniformity, conductive transparent single-walled-carbon-nanotube films for 
photovoltaics produced by ultrasonic spraying $A d v$. Mater. 21 3210-6

[23] Geng H-Z, Kim K K, So K P, Lee Y S, Chang Y and Lee Y H 2007 Effect of acid treatment on carbon nanotube-based flexible transparent conducting films J. Am. Chem. Soc. $1297758-9$

[24] Hecht D S, Heintz A M, Lee R, Hu L, Moore B, Cucksey C and Risser S 2011 High conductivity transparent carbon nanotube films deposited from superacid Nanotechnology 22075201

[25] Shin D-W, Lee J H, Kim Y-H, Yu S M, Park S-Y and Yoo J-B 2009 A role of $\mathrm{HNO}_{3}$ on transparent conducting film with single-walled carbon nanotubes Nanotechnology 20475703

[26] Shim B S, Zhu J, Jan E, Critchley K and Kotov N A 2010 Transparent conductors from layer-by-layer assembled SWNT films: importance of mechanical properties and a new figure of merit ACS Nano 4 3725-34

[27] Lee J-Y, Connor S T, Cui Y and Peumans P 2008 Solution-processed metal nanowire mesh transparent electrodes Nano Lett. 8 689-92

[28] Madaria A, Kumar A, Ishikawa F and Zhou C 2010 Uniform, highly conductive, and patterned transparent films of a percolating silver nanowire network on rigid and flexible substrates using a dry transfer technique Nano Res. 3 564-73

[29] Lu Y C and Chou K S 2010 Tailoring of silver wires and their performance as transparent conductive coatings Nanotechnology 21215707

[30] Scardaci V, Coull R, Lyons P E, Rickard D and Coleman J N 2011 Spray deposition of highly transparent, low-resistance networks of silver nanowires over large areas Small 7 2621-8

[31] Tokuno T, Nogi M, Karakawa M, Jiu J, Nge T, Aso Y and Suganuma K 2011 Fabrication of silver nanowire transparent electrodes at room temperature Nano Res. 4 1215-22

[32] Garnett E C, Cai W, Cha J J, Mahmood F, Connor S T, Greyson Christoforo M, Cui Y, McGehee M D and Brongersma M L 2012 Self-limited plasmonic welding of silver nanowire junctions Nature Mater. 11 241-9

[33] Preston C, Xu Y,Han X, Munday J N and Hu L 2013 Optical haze of transs $\equiv$ and conductive silver nanowire films Nano Res. at press

[34] Emmott C J M, Urbina A and Nelson J 2012 Environmental and economic assessment of ITO-free electrodes for organic solar cells Sol. Energy Mater. Sol. Cells 97 14-21

[35] Rathmell A R, Bergin S M, Hua Y-L, Li Z-Y and Wiley B J 2010 The growth mechanism of copper nanowires and their properties in flexible, transparent conducting films Adv. Mater. 22 3558-63

[36] Rathmell A R and Wiley B J 2011 The synthesis and coating of long, thin copper nanowires to make flexible, transparent conducting films on plastic substrates $A d v$. Mater. 23 4798-803

[37] Wu H, Hu L, Rowell M W, Kong D, Cha J J, McDonough J R, Zhu J, Yang Y, McGehee M D and Cui Y 2010 Electrospun metal nanofiber webs as high-performance transparent electrode Nano Lett. $104242-8$

[38] Zhang D, Wang R, Wen M, Weng D, Cui X, Sun J, Li H and $\mathrm{Lu} \mathrm{Y} 2012$ Synthesis of ultralong copper nanowires for high-performance transparent electrodes J. Am. Chem. Soc. 134 14283-6

[39] Lyons P E, De S, Elias J, Schamel M, Philippe L, Bellew A T, Boland J J and Coleman J N 2011
High-performance transparent conductors from networks of gold nanowires J. Phys. Chem. Lett. 2 3058-62

[40] Sánchez-Iglesias A, Rivas-Murias B, Grzelczak M, Pérez-Juste J, Liz-Marzán L M, Rivadulla F and Correa-Duarte M A 2012 Highly transparent and conductive films of densely aligned ultrathin Au nanowire monolayers Nano Lett. 12 6066-70

[41] Rathmell A R, Nguyen M, Chi M and Wiley B J 2012 Synthesis of oxidation-resistant cupronickel nanowires for transparent conducting nanowire networks Nano Lett. 12 3193-9

[42] Coskun S, Aksoy B and Unalan H E 2011 Polyol synthesis of silver nanowires: an extensive parametric study Cryst. Growth Des. 11 4963-9

[43] Chen D, Qiao X and Chen J 2011 Morphology-controlled synthesis of silver nanostructures via a solvothermal method J. Mater. Sci.: Mater. Electron. 22 1335-9

[44] Fiévet F, Lagier J P and Figlarz M 1989 Preparing monodisperse metal powders in micrometer and submicrometer size by the polyol process MRS Bull. 1429

[45] Hu M, Gao J, Dong Y, Yang S and Li R K Y 2012 Rapid controllable high-concentration synthesis and mutual attachment of silver nanowires RSC Adv. 2 2055-60

[46] Sun Y, Mayers B, Herricks T and Xia Y 2003 Polyol synthesis of uniform silver nanowires: a plausible growth mechanism and the supporting evidence Nano Lett. 3 955-60

[47] Sun Y, Gates B, Mayers B and Xia Y 2002 Crystalline silver nanowires by soft solution processing Nano Lett. 2 165-8

[48] Sun Y and Xia Y 2002 Large-scale synthesis of uniform silver nanowires through a soft, self-seeding, polyol process Adv. Mater. 14 833-7

[49] Xia Y, Xiong Y, Lim B and Skrabalak S E 2009 Shape-controlled synthesis of metal nanocrystals: simple chemistry meets complex physics? Angew. Chem. Int. Edn 48 60-103

[50] Chen C, Wang L, Jiang G, Yang Q, Wang J, Yu H, Chen T, Wang $\mathrm{C}$ and Chen X 2006 The influence of seeding conditions and shielding gas atmosphere on the synthesis of silver nanowires through the polyol process Nanotechnology 17 466-74

[51] Gao Y et al 2005 Growth mechanism of silver nanowires synthesized by polyvinylpyrrolidone-assisted polyol reduction J. Phys. D: Appl. Phys 38 1061-7

[52] Hu L, Kim H S, Lee J-Y, Peumans P and Cui Y 2010 Scalable coating and properties of transparent, flexible, silver nanowire electrodes ACS Nano 4 2955-63

[53] Chang S, Chen K, Hua Q, Ma Y and Huang W 2011 Evidence for the growth mechanisms of silver nanocubes and nanowires J. Phys. Chem. C 115 7979-86

[54] Wiley B, Sun Y and Xia Y 2005 Polyol synthesis of silver nanostructures: control of product morphology with $\mathrm{Fe}(\mathrm{II})$ or Fe(III) species Langmuir 21 8077-80

[55] Korte K E, Skrabalak S E and Xia Y 2008 Rapid synthesis of silver nanowires through a CuCl- or $\mathrm{CuCl}_{2}$-mediated polyol process J. Mater. Chem. 18 437-41

[56] Wiley B, Sun Y, Mayers B and Xia Y 2005 Shape-controlled synthesis of metal nanostructures: the case of silver Chem.-Eur. J. 11 454-63

[57] Sun Y, Ren Y, Liu Y, Wen J, Okasinski J S and Miller D J 2012 Ambient-stable tetragonal phase in silver nanostructures Nature Commun. 3971

[58] Gao Y et al 2004 Evidence for the monolayer assembly of poly(vinylpyrrolidone) on the surfaces of silver nanowires J. Phys. Chem. B 108 12877-81

[59] Celle C, Mayousse C, Moreau E, Basti H, Carella A and Simonato J-P 2012 Highly flexible transparent film heaters 
based on random networks of silver nanowires Nano Res. 5 427-33

[60] Lee J H, Lee P, Lee D, Lee S S and Ko S H 2012 Large-scale synthesis and characterization of very long silver nanowires via successive multistep growth Cryst. Growth Des. 12 5598-605

[61] Sun Y, Yin Y, Mayers B T, Herricks T and Xia Y 2002 Uniform silver nanowires synthesis by reducing $\mathrm{AgNO}_{3}$ with ethylene glycol in the presence of seeds and poly(vinyl pyrrolidone) Chem. Mater. 14 4736-45

[62] Zhang W C, Wu X L, Chen H T, Gao Y J, Zhu J, Huang G S and Chu P K 2008 Self-organized formation of silver nanowires, nanocubes and bipyramids via a solvothermal method Acta Mater. 56 2508-13

[63] Wiley B, Herricks T, Sun Y and Xia Y 2004 Polyol synthesis of silver nanoparticles: use of chloride and oxygen to promote the formation of single-crystal, truncated cubes and tetrahedrons Nano Lett. 4 1733-9

[64] Gou L, Chipara M and Zaleski J M 2007 Convenient, rapid synthesis of Ag nanowires Chem. Mater. 19 1755-60

[65] Luu Q N, Doorn J M, Berry M T, Jiang C, Lin C and May P S 2011 Preparation and optical properties of silver nanowires and silver-nanowire thin films $J$. Colloid Interface Sci. 356 151-8

[66] Kim T, Canlier A, Kim G H, Choi J, Park M and Han S M 2013 Electrostatic spray deposition of highly transparent silver nanowire electrode on flexible substrate ACS Appl. Mater. Interfaces 5 788-94

[67] Lee J, Lee I $\mathrm{Kin}$ T-S and Lee J-Y 2013 Efficient welding of silver nat $\bar{\equiv}$ e networks without post-processing Small at press

[68] Pradel K C, Sohn K and Huang J 2011 Cross-flow purification of nanowires Angew. Chem. Int. Edn $503412-6$

[69] Mayousse C, Celle C, Moreau E, Mainguet J-F, Carella A and Simonato J-P 2013 Improvements in purification of silver nanowires by decantation and fabrication of flexible transparent electrodes. Application to capacitive touch sensors Nanotechnology 24215501

[70] Catrysse P B and Fan S 2010 Nanopatterned metallic films for use as transparent conductive electrodes in optoelectronic devices Nano Lett. $102944-9$

[71] Lee J-Y, Connor S T, Cui Y and Peumans P 2010 Semitransparent organic photovoltaic cells with laminated top electrode Nano Lett. 10 1276-9

[72] Hardin B E, Gaynor W, Ding I-K, Rim S-B, Peumans P and McGehee M D 2011 Laminating solution-processed silver nanowire mesh electrodes onto solid-state dye-sensitized solar cells Org. Electron. 12 875-9

[73] Chung C-H, Song T-B, Bob B, Zhu R and Yang Y 2012 Solution-processed flexible transparent conductors composed of silver nanowire networks embedded in indium tin oxide nanoparticle matrices Nano Res. 5 805-14

[74] Leem D-S, Edwards A, Faist M, Nelson J, Bradley D D C and de Mello J C 2011 Efficient organic solar cells with solution-processed silver nanowire electrodes Adv. Mater. 23 4371-5

[75] Liu C-H and Yu X 2011 Silver nanowire-based transparent, flexible, and conductive thin film Nanoscale Res. Lett. 6 75-82

[76] Deegan R D, Bakajin O, Dupont T F, Huber G, Nagel S R and Witten T A 1997 Capillary flow as the cause of ring stains from dried liquid drops Nature 389 827-9

[77] Zhou Y, Chen J, Zhang L and Yang L 2012 Multifunctional $\mathrm{TiO}_{2}$-coated Ag nanowire arrays as recyclable SERS substrates for the detection of organic pollutants Eur. $J$. Inorg. Chem. 2012 3176-82

[78] Al-Mawlawi D, Liu C Z and Moskovits M 1994 Nanowires formed in anodic oxide nanotemplates J. Mater. Res. 9 1014-8

[79] Madaria A R, Kumar A and Zhou C 2011 Large scale, highly conductive and patterned transparent films of silver nanowires on arbitrary substrates and their application in touch screens Nanotechnology 22245201

[80] Yu G, Cao A and Lieber C M 2007 Large-area blown bubble films of aligned nanowires and carbon nanotubes Nature Nanotechnol. 2 372-7

[81] Huang J, Fan R, Connor S and Yang P 2007 One-step patterning of aligned nanowire arrays by programmed dip coating Angew. Chem. Int. Edn 46 2414-7

[82] Whang D, Jin S, Wu Y and Lieber C M 2003 Large-scale hierarchical organization of nanowire arrays for integrated nanosystems Nano Lett. 3 1255-9

[83] Salalha W and Zussman E 2005 Investigation of fluidic assembly of nanowires using a droplet inside microchannels Phys. Fluids 17063301

[84] Huang Y, Duan X, Wei Q and Lieber C M 2001 Directed assembly of one-dimensional nanostructures into functional networks Science 291 630-3

[85] Smith P A, Nordquist C D, Jackson T N, Mayer T S, Martin B R, Mbindyo J and Mallouk T E 2000 Electric-field assisted assembly and alignment of metallic nanowires Appl. Phys. Lett. 77 1399-401

[86] Harnack O, Pacholski C, Weller H, Yasuda A and Wessels J M 2003 Rectifying behavior of electrically aligned ZnO nanorods Nano Lett. 3 1097-101

[87] Sun B and Sirringhaus H 2006 Surface tension and fluid flow driven self-assembly of ordered $\mathrm{ZnO}$ nanorod films for high-performance field effect transistors J. Am. Chem. Soc. 128 16231-7

[88] Cui Y, Björk M T, Liddle J A, Sönnichsen C, Boussert B and Alivisatos A P 2004 Integration of colloidal nanocrystals into lithographically patterned devices Nano Lett. 4 1093-8

[89] Yin Y, Lu Y, Gates B and Xia Y 2001 Template-assisted self-assembly: a practical route to complex aggregates of monodispersed colloids with well-defined sizes, shapes, and structures J. Am. Chem. Soc. 123 8718-29

[90] Engel M, Small J P, Steiner M, Freitag M, Green A A, Hersam M C and Avouris P 2008 Thin film nanotube transistors based on self-assembled, aligned, semiconducting carbon nanotube arrays ACS Nano 2 2445-52

[91] Heo K et al 2008 Large-scale assembly of silicon nanowire network-based devices using conventional microfabrication facilities Nano Lett. 8 4523-7

[92] Rao S G, Huang L, Setyawan W and Hong S 2003 Nanotube electronics: large-scale assembly of carbon nanotubes Nature 425 36-7

[93] Javey A, Nam S W, Friedman R S, Yan H and Lieber C M 2007 Layer-by-layer assembly of nanowires for three-dimensional, multifunctional electronics Nano Lett. $7773-7$

[94] Seo J, Lee H, Lee S, Lee T I, Myoung J-M and Lee T 2012 Direct gravure printing of silicon nanowires using entropic attraction forces Small 8 1614-21

[95] Hsu P-C, Wu H, Carney T J, McDowell M T, Yang Y, Garnett E C, Li M, Hu L and Cui Y 2012 Passivation coating on electrospun copper nanofibers for stable transparent electrodes ACS Nano 6 5150-6

[96] Gaynor W, Burkhard G F, McGehee M D and Peumans P 2011 Smooth nanowire/polymer composite transparent electrodes Adv. Mater. 23 2905-10 
[97] Zeng X-Y, Zhang Q-K, Yu R-M and Lu C-Z 2010 A new transparent conductor: silver nanowire film buried at the surface of a transparent polymer Adv. Mater. 22 4484-8

[98] Kim A, Won Y, Woo K, Kim C-H and Moon J 2013 Highly transparent low resistance $\mathrm{ZnO} / \mathrm{Ag}$ nanowire/ZnO composite electrode for thin film solar cells ACS Nano 7 1081-91

[99] Morgenstern F S F, Kabra D, Massip S, Brenner T J K, Lyons P E, Coleman J N and Friend R H 2011 Ag-nanowire films coated with $\mathrm{ZnO}$ nanoparticles as a transparent electrode for solar cells Appl. Phys. Lett. 99183307

[100] Ramasamy P, Seo D-M, Kim S-H and Kim J 2012 Effects of $\mathrm{TiO}_{2}$ shells on optical and thermal properties of silver nanowires J. Mater. Chem. 22 11651-7

[101] Sorel S, Lyons P E, De S, Dickerson J C and Coleman J N 2012 The dependence of the optoelectrical properties of silver nanowire networks on nanowire length and diameter Nanotechnology 23185201

[102] Bid A, Bora A and Raychaudhuri A K 2006 Temperature dependence of the resistance of metallic nanowires of diameter $\geq 15 \mathrm{~nm}$ : applicability of Bloch-Grüneisen theorem Phys. Rev. B 74035426

[103] Hecht D, Hu L and Grüner G 2006 Conductivity scaling with bundle length and diameter in single walled carbon nanotube networks Appl. Phys. Lett. 89133112

[104] Zhao J, Sun H, Dai S, Wang Y and Zhu J 2011 Electrical breakdown of nanowires Nano Lett. 11 4647-51

[105] Quinten M 2011 Front matter Optical Properties of Nanoparticle Systems: Mie and Beyond Optical Properties of Nanoparticle Systems (New York: Wiley-VCH) pp 1-14

[106] Ritchie R H 1957 Plasma losses by fast electrons in thin films Phys. Rev. 106 874-81

[107] Schaffer B, Hohenester U, Trügler A and Hofer F 2009 High-resolution surface plasmon imaging of gold nanoparticles by energy-filtered transmission electron microscopy Phys. Rev. B 79041401

[108] Powell C J and Swan J B 1959 Origin of the characteristic electron energy losses in aluminum Phys. Rev. 115 869-75

[109] Ditlbacher H, Hohenau A, Wagner D, Kreibig U, Rogers M, Hofer F, Aussenegg F R and Krenn J R 2005 Silver nanowires as surface plasmon resonators Phys. Rev. Lett. 95257403

[110] Kenens B, Rybachuk M, Hofkens J and Uji-i H 2013 Silver nanowires terminated by metallic nanoparticles as effective plasmonic antennas J. Phys. Chem. C 117 2547-53

[111] Kottmann J, Martin O, Smith D and Schultz S 2000 Spectral response of plasmon resonant nanoparticles with a non-regular shape Opt. Express 6 213-9

[112] Kottmann J P, Martin O J F, Smith D R and Schultz S 2001 Plasmon resonances of silver nanowires with a nonregular cross section Phys. Rev. B 64235402

[113] Spechler J A and Arnold C B 2012 Direct-write pulsed laser processed silver nanowire networks for transparent conducting electrodes Appl. Phys. A 108 25-8

[114] $\mathrm{Hu} \mathrm{L}$ and Chen G 2007 Analysis of optical absorption in silicon nanowire arrays for photovoltaic applications Nano Lett. 7 3249-52

[115] Chih-Hung T et al 2011 Influences of textures in fluorine-doped tin oxide on characteristics of dye-sensitized solar cells Org. Electron. 12 2003-11

[116] Guillén C, Montero J and Herrero J 2013 Transparent and conductive electrodes combining AZO and ATO thin films for enhanced light scattering and electrical performance Appl. Surf. Sci. 264 448-52
[117] Kuramochi H, Akiike R, Iigusa H, Tamano K, Utsumi K, Shibutami T, Sichanugrist P and Konagai M 2012 Development of novel aluminum-doped zinc oxide film and its application to solar cells Japan. J. Appl. Phys. 51 10NB13

[118] De S and Coleman J N 2011 The effects of percolation in nanostructured transparent conductors MRS Bull. $36774-81$

[119] Haacke G 1976 New figure of merit for transparent conductors J. Appl. Phys. 47 4086-9

[120] Chen Y-Y, Chen C-L Le P-C and Ou M-N 2011 Fabrication, char $\equiv$ zation and thermal properties of nanowires Nanowires-Fundamental Research ed A Hashim, InTech

[121] Völklein F, Reith H, Cornelius T W, Rauber M and Neumann R 2009 The experimental investigation of thermal conductivity and the Wiedemann-Franz law for single metallic nanowires Nanotechnology 20325706

[122] Peng Y, Cullis T and Inkson B 2008 Accurate electrical testing of individual gold nanowires by in situ scanning electron microscope nanomanipulators Appl. Phys. Lett. 93183112

[123] Zeng J L, Cao Z, Yang D W, Sun L X and Zhang L 2009 Thermal conductivity enhancement of Ag nanowires on an organic phase change material J. Therm. Anal. Calorim. $101385-9$

[124] Xu J, Munari A, Dalton E, Mathewson A and Razeeb K M 2009 Silver nanowire array-polymer composite as thermal interface material J. Appl. Phys. 106124310

[125] Reynolds F W and Stilwell G R 1952 Mean free paths of electrons in evaporated metal films Phys. Rev. 88 418-9

[126] Dingle R B 1950 The electrical conductivity of thin wires Proc. R. Soc. A 201 545-60

[127] Rayleigh L 1878 On the instability of jets Proc. Lond. Math. Soc. s1-10 4-13

[128] Karim S, Toimil-Molares M E, Balogh A G, Ensinger W, Cornelius T W, Khan E U and Neumann R 2006 Morphological evolution of Au nanowires controlled by Rayleigh instability Nanotechnology 17 5954-9

[129] Li H, Biser J M, Perkins J T, Dutta S, Vinci R P and Chan H M 2008 Thermal stability of $\mathrm{Cu}$ nanowires on a sapphire substrate J. Appl. Phys. 103024315

[130] Huang R, Wen Y-H, Zhu Z-Z and Sun S-G 2011 Thermal stability of platinum nanowires: a comparison study between single-crystalline and twinned structures J. Mater. Chem. 21 18998-9004

[131] Shin H S, Yu J and Song J Y 2007 Size-dependent thermal instability and melting behavior of Sn nanowires Appl. Phys. Lett. 91173106

[132] Hsiung C-P, Liao H-W, Gan J-Y, Wu T-B, Hwang J-C, Chen F and Tsai M-J 2010 Formation and instability of silver nanofilament in Ag-based programmable metallization cells ACS Nano 4 5414-20

[133] Yu Z, Zhang Q, Li L, Chen Q, Niu X, Liu J and Pei Q 2011 Highly flexible silver nanowire electrodes for shape-memory polymer light-emitting diodes Adv. Mater. 23 664-8

[134] Lim J-W, Cho D-Y, Eun K, Choa S-H, Na S-I, Kim J and Kim H-K 2012 Mechanical integrity of flexible Ag nanowire network electrodes coated on colorless PI substrates for flexible organic solar cells Sol. Energy Mater. Sol. Cells 105 69-76

[135] Consonni V, Rey G, Roussel H, Doisneau B, Blanquet E and Bellet D 2013 Preferential orientation of fluorine-doped $\mathrm{SnO}_{2}$ thin films: the effects of growth temperature Acta Mater. 61 22-31

[136] Lee P, Lee J, Lee H, Yeo J, Hong S, Nam K H, Lee D, Lee S S and Ko S H 2012 Highly stretchable and highly 
conductive metal electrode by very long metal nanowire percolation network Adv. Mater. 24 3326-32

[137] Xu F and Zhu Y 2012 Highly conductive and stretchable silver nanowire conductors Adv. Mater. 24 5117-22

[138] Ho X, Nie Tey J, Liu W, Kweng Cheng C and Wei J 2013 Biaxially stretchable silver nanowire transparent conductors J. Appl. Phys. 113044311

[139] Balberg I, Binenbaum N and Anderson C H 1983 Critical behavior of the two-dimensional sticks system Phys. Rev. Lett. 51 1605-8

[140] Li J and Zhang S-L 2009 Finite-size scaling in stick percolation Phys. Rev. E 80040104

[141] Žeželj M, Stanković I and Belić A 2012 Finite-size scaling in asymmetric systems of percolating sticks Phys. Rev. E 85021101

[142] Newman M E J and Ziff R M 2001 Fast Monte Carlo algorithm for site or bond percolation Phys. Rev. E 64016706

[143] Hovi J-P and Aharony A 1996 Scaling and universality in the spanning probability for percolation Phys. Rev. E $53235-53$

[144] White S I, DiDonna B A, Mu M, Lubensky T C and Winey K I 2009 Simulations and electrical conductivity of percolated networks of finite rods with various degrees of axial alignment Phys. Rev. B 79024301

[145] Žeželj M and Stanković I 2012 From percolating to dense random stick networks: conductivity model investigation Phys. Rev. B 86134202

[146] De S, King P J, Lyons P E, Khan U and Coleman J N 2010 Size effects and the problem with percolation in nanostructured transparent conductors ACS Nano 4 7064-72

[147] Nirmalraj P N et al 2012 Manipulating connectivity and electrical conductivity in metallic nanowire networks Nano Lett. 12 5966-71

[148] Mie G 1908 Beiträge zur optik trüber medien, speziell kolloidaler metallösungen Ann. Phys., Lpz. 330 377-445

[149] Li L 1997 New formulation of the Fourier modal method for crossed surface-relief gratings J. Opt. Soc. Am. A $142758-67$

[150] Kottmann J P and Martin O J F 2000 Accurate solution of the volume integral equation for high-permittivity scatterers IEEE Trans. Antennas Propag. 48 1719-26

[151] Waterman P C 1965 Matrix formulation of electromagnetic scattering Proc. IEEE 53 805-12

[152] Barber P W 1977 Resonance electromagnetic absorption by nonspherical dielectric objects IEEE Trans. Microw. Theory Tech. 25 373-81

[153] Mishchenko M I, Travis L D and Lacis A A 2002 Scattering, Absorption, and Emission of Light by Small Particles (Cambridge: Cambridge University Press)

[154] Larciprete M C et al 2012 Infrared properties of randomly oriented silver nanowires J. Appl. Phys. 112083503

[155] Aden A L and Kerker M 1951 Scattering of electromagnetic waves from two concentric spheres J. Appl. Phys. 22 1242-6

[156] Taflove A and Hagness S C 2005 Computational Electrodynamics: The Finite-Difference Time-Domain Method (Boston, MA: Artech House Publishers)

[157] Volakis J L, Chatterjee A and Kempel L C 1994 Review of the finite-element method for three-dimensional electromagnetic scattering J. Opt. Soc. Am. A 11 1422-33

[158] Rother T and Schmidt K 1996 The discretized Mie-formalism for plane wave scattering on dielectric objects with non-separable geometries J. Quant. Spectrosc. Radiat. Transfer 55 615-25
[159] Rother T, Schmidt K and Wauer J 1997 Plane wave scattering on hexagonal cylinders J. Quant. Spectrosc. Radiat. Transfer 57 669-81

[160] Purcell E M and Pennypacker C R 1973 Scattering and absorption of light by nonspherical dielectric grains Astrophys. J. 186 705-14

[161] Bonner R F, Nossal R, Havlin S and Weiss G H 1987 Model for photon migration in turbid biological media J. Opt. Soc. Am. A 4 423-32

[162] Lalanne P and Jurek M P 1998 Computation of the near-field pattern with the coupled-wave method for transverse magnetic polarization J. Mod. Opt. 45 1357-74

[163] Hao E and Schatz G C 2004 Electromagnetic fields around silver nanoparticles and dimers J. Chem. Phys. $120357-66$

[164] Gandjbakhche A H, Nossal R and Bonner R F 1993 Scaling relationships for theories of anisotropic random walks applied to tissue optics Appl. Opt. 32 504-16

[165] Yurkin M A and Hoekstra A G 2007 The discrete dipole approximation: an overview and recent developments J. Quant. Spectrosc. Radiat. Transfer 106 558-89

[166] Yee K 1966 Numerical solution of initial boundary value problems involving Maxwell's equations in isotropic media IEEE Trans. Antennas Propag. 14 302-7

[167] Gandjbakhche A H, Weiss G H, Bonner R F and Nossal R 1993 Photon path-length distributions for transmission through optically turbid slabs Phys. Rev. E 48 810-8

[168] Brönstrup G, Garwe F, Csáki A, Fritzsche W, Steinbrück A and Christiansen S 2011 Statistical model on the optical properties of silicon nanowire mats Phys. Rev. B 84125432

[169] Street R A, Wong W S and Paulson C 2009 Analytic model for diffuse reflectivity of silicon nanowire mats Nano Lett. $93494-7$

[170] Li L, Yu Z, Hu W, Chang C, Chen Q and Pei Q 2011 Efficient flexible phosphorescent polymer light-emitting diodes based on silver nanowire-polymer composite electrode Adv. Mater. 23 5563-7

[171] Gaynor W, Lee J-Y and Peumans P 2010 Fully solution-processed inverted polymer solar cells with laminated nanowire electrodes ACS Nano 4 30-4

[172] Sun T, Wang Z L, Shi Z J, Ran G Z, Xu W J, Wang Z Y, Li Y Z, Dai L and Qin G G 2010 Multilayered graphene used as anode of organic light emitting devices Appl. Phys. Lett. 96133301

[173] Yang L, Zhang T, Zhou H, Price S C, Wiley B J and You W 2011 Solution-processed flexible polymer solar cells with silver nanowire electrodes ACS Appl. Mater. Interfaces 3 4075-84

[174] Chen C-C, Dou L, Zhu R, Chung C-H, Song T-B, Zheng Y B, Hawks S, Li G, Weiss P S and Yang Y 2012 Visibly transparent polymer solar cells produced by solution processing ACS Nano $67185-90$

[175] Ajuria J, Ugarte I, Cambarau W, Etxebarria I, Tena-Zaera R and Pacios R 2012 Insights on the working principles of flexible and efficient ITO-free organic solar cells based on solution processed Ag nanowire electrodes Sol. Energy Mater. Sol. Cells 102 148-52

[176] Stubhan T, Krantz J, Li N, Guo F, Litzov I, Steidl M, Richter M, Matt G J and Brabec C J 2012 High fill factor polymer solar cells comprising a transparent, low temperature solution processed doped metal oxide/metal nanowire composite electrode Sol. Energy Mater. Sol. Cells 107 248-51

[177] Kim T, Kim Y W, Lee H S, Kim H, Yang W S and Suh K S 2013 Uniformly interconnected silver-nanowire networks for transparent film heaters Adv. Funct. Mater. 23 1250-5

[178] Simonato J-P, Celle C, Mayousse C, Carella A, Basti H and Carpentier A 2012 Transparent film heaters based on 
silver nano $\Rightarrow$ random networks MRS Online Proc. Lib. 1449 107-

[179] Müllen K and Scherf U 2006 Organic Light Emitting Devices: Synthesis, Properties and Applications (New York: Wiley-VCH)

[180] Li L, Yu Z, Chang C, Hu W, Niu X, Chen Q and Pei Q 2012 Efficient white polymer light-emitting diodes employing a silver nanowire-polymer composite electrode Phys. Chem. Chem. Phys. 14 14249-54

[181] Gaynor W et al 2013 Color in the corner $\equiv$-free white OLEDs with angular color stability AdV.Mater. at press

[182] Kim K-K, Lee S, Kim H, Park J-C, Lee S-N, Park Y, Park S-J and Kim S-W 2009 Enhanced light extraction efficiency of
GaN-based light-emitting diodes with $\mathrm{ZnO}$ nanorod arrays grown using aqueous solution Appl. Phys. Lett. 94071118

[183] Coskun S, Selen Ates E and Emrah Unalan H 2013

Optimization of silver nanowire networks for polymer light emitting diode electrodes Nanotechnology 24125202

[184] Lee J, Lee P, Lee H, Lee D, Lee S S and Ko S H 2012 Very long Ag nanowire synthesis and its application in a highly transparent, conductive and flexible metal electrode touch panel Nanoscale 4 6408-14

[185] Hu W, Niu X, Zhao R and Pei Q 2013 Elastomeric transparent capacitive sensors based on an interpenetrating composite of silver nanowires and polyurethane Appl. Phys. Lett. 102083303 


\section{Queries for IOP paper 469533}

Journal: $\quad$ Nano

Author: D Langley et al

Short title: Flexible transparent conductive materials

based on silver nanowire networks: a review

Page 1

Query 1:

Author: Please check that the author names and affiliations as given are spelt correctly.

Page 1

Query 2:

Author: Please be aware that the colour figures in this article will only appear in colour in the Web version. If you require colour in the printed journal and have not previously arranged it, please contact the Production Editor now.

\section{Page 6}

Query 3:

Author: An extra closing parenthesis is deleted in figure 4. Please check.

\section{Page 12}

Query 4:

Author: is 'open square box' here and throughout ok (or is it due to file corruption)?

\section{Page 15}

Query 5:

Author: Please check the details for any journal references that do not have a blue link as they may contain some incorrect information. Pale purple links are used for references to arXiv e-prints.

\section{Page 16}

Query 6:

Author: [33, 67, 181]: Any update?

\section{Page 18}

Query 7:

Author: [110]: Please check last author name given here.

\section{Page 18}

Query 8:

Author: [120]: Please provide place of publisher.

\section{Page 20}

Query 9:

Author: [178]: Please check the journal title given here. 